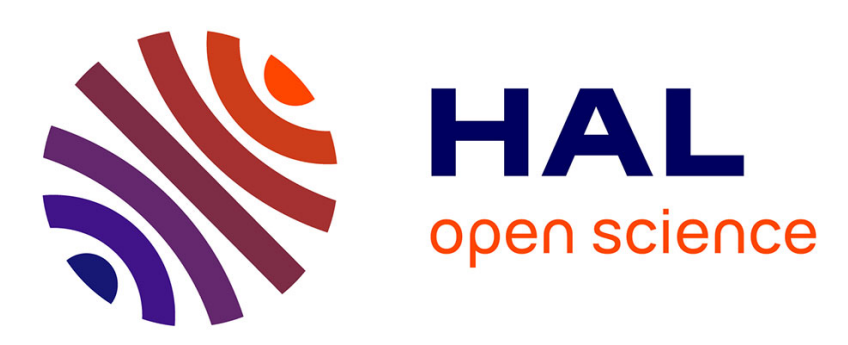

\title{
Numerical analysis of nodal sets for eigenvalues of Aharonov-Bohm Hamiltonians on the square and application to minimal partitions
}

Virginie Bonnaillie-Noël, Bernard Helffer

\section{- To cite this version:}

Virginie Bonnaillie-Noël, Bernard Helffer. Numerical analysis of nodal sets for eigenvalues of Aharonov-Bohm Hamiltonians on the square and application to minimal partitions. Experimental Mathematics, 2011, 20 (3), pp.304-322. 10.1080/10586458.2011.565240 . hal-00429551

\section{HAL Id: hal-00429551 \\ https://hal.science/hal-00429551}

Submitted on 3 Nov 2009

HAL is a multi-disciplinary open access archive for the deposit and dissemination of scientific research documents, whether they are published or not. The documents may come from teaching and research institutions in France or abroad, or from public or private research centers.
L'archive ouverte pluridisciplinaire HAL, est destinée au dépôt et à la diffusion de documents scientifiques de niveau recherche, publiés ou non, émanant des établissements d'enseignement et de recherche français ou étrangers, des laboratoires publics ou privés. 


\title{
Numerical analysis of nodal sets for eigenvalues of Aharonov-Bohm Hamiltonians on the square and application to minimal partitions
}

\author{
V. Bonnaillie-Noël* and B. Helffer ${ }^{\dagger}$
}

October 29, 2009

\begin{abstract}
Using the double covering approach introduced by B. Helffer, M. and T. HoffmannOstenhof and M. Owen and further developed for questions of isospectrality by the authors in collaboration with T. Hoffmann-Ostenhof, we analyze the variation of the eigenvalues of the one pole Aharonov-Bohm Hamiltonian on the square and the nodal picture of the associated eigenfunctions as a function of the pole. This leads us to discover new candidates for minimal $k$-partitions of the square with a specific topological type. This illustrates also recent results of B. Noris and S. Terracini. This finally supports or disproves conjectures for the minimal 3 and 5-partitions on the square.
\end{abstract}

\section{Introduction}

The recent papers [12] and [3] have shown the strong links between the analysis of nodal sets of some eigenfunctions of Aharonov-Bohm Hamiltonians and the question of minimal partitions. In connection with recent papers of B. Noris and S. Terracini [18, 19], we analyze the dependence of the nodal picture of the eigenvalues of the Aharonov-Bohm Hamiltonian on the square as a function of the pole and propose new candidates for minimal $k$-partitions of a specific topological type for the square.

\subsection{Aharonov-Bohm Hamiltonian}

Let us recall some definitions and results about the Aharonov-Bohm Hamiltonian (for short $\mathbf{A B} X$-Hamiltonian) with a singularity at $X$ introduced in $[3,10]$ and motivated by the work of Berger-Rubinstein [2]. We denote by $X=\left(x_{0}, y_{0}\right)$ the coordinates of the pole and consider the magnetic potential with flux $\Phi=1 / 2$ :

$$
\mathbf{A}^{X}(x, y)=\left(A_{1}^{X}(x, y), A_{2}^{X}(x, y)\right)=\frac{1}{2}\left(-\frac{y-y_{0}}{r^{2}}, \frac{x-x_{0}}{r^{2}}\right) .
$$

\footnotetext{
*IRMAR, ENS Cachan Bretagne, Univ. Rennes 1, CNRS, UEB, av. Robert Schuman, F-35170 Bruz, France, Virginie.Bonnaillie@bretagne.ens-cachan.fr

${ }^{\dagger}$ Laboratoire de Mathématiques, Bat. 425, Univ Paris-Sud and CNRS, F-91405 Orsay Cedex, France, Bernard.Helffer@math.u-psud.fr
} 
We know that the magnetic field vanishes identically in $\dot{\Omega}_{X}$. The $\mathbf{A B} X$-Hamiltonian is defined by considering the Friedrichs extension starting from $C_{0}^{\infty}\left(\dot{\Omega}_{X}\right)$ and the associated differential operator is

$$
-\Delta_{\mathbf{A}^{X}}:=\left(D_{x}-A_{1}^{X}\right)^{2}+\left(D_{y}-A_{2}^{X}\right)^{2},
$$

with $D_{x}=-i \partial_{x}$ and $D_{y}=-i \partial_{y}$.

This operator is preserving the $K_{X}$-real functions in the following sense. We say that a function $u$ is $K_{X}$-real, if it satisfies $K_{X} u=u$, where $K_{X}$ is the antilinear operator

$$
K_{X}=e^{i \theta_{X}} \Gamma,
$$

with $\left(x-x_{0}\right)+i\left(y-y_{0}\right)=\sqrt{\left|x-x_{0}\right|^{2}+\left|y-y_{0}\right|^{2}} e^{i \theta_{X}}$, and where $\Gamma$ is the complex conjugation operator $\Gamma u=\bar{u}$.

Then $-\Delta_{\mathbf{A}^{X}}$ preserves $K_{X^{-}}$-real functions and we can consider a basis of $K_{X^{-}}$-real eigenfunctions. Hence we only analyze the restriction of the $\mathbf{A B} X$-Hamiltonian to the $K_{X}$-real space $L_{K_{X}}^{2}$ where

$$
L_{K_{X}}^{2}\left(\dot{\Omega}_{X}\right)=\left\{u \in L^{2}\left(\dot{\Omega}_{X}\right), K_{X} u=u\right\} .
$$

When no ambiguity exists, we omit sometimes the reference to $X$ and write more simply $\theta, K, L_{K}^{2},-\Delta_{\mathbf{A}}$.

\subsection{Minimal partitions}

For a given partition $\mathcal{D}$ of an open set $\Omega$ by $k$ disjoint open subsets $D_{i}$, we consider

$$
\Lambda(\mathcal{D})=\max _{i=1, \ldots, k} \lambda\left(D_{i}\right)
$$

where $\lambda\left(D_{i}\right)$ is the groundstate energy of the Dirichlet Laplacian on $D_{i}$. We denote the infimum of $\Lambda$ over all $k$-partitions of $\Omega$ by

$$
\mathfrak{L}_{k}(\Omega)=\inf _{\mathcal{D} \in \mathfrak{O}_{k}} \Lambda(\mathcal{D}) .
$$

We look for minimal $k$-partitions, that are partitions $\mathcal{D}=\left(D_{1}, \ldots, D_{k}\right)$, such that

$$
\mathfrak{L}_{k}(\Omega)=\Lambda(\mathcal{D}) .
$$

We recall that these minimal $k$-partitions, whose existence was proven in $[6,8,7]$, share with nodal domains many properties of regularity, except that the number of half-lines meeting (with equal angle) at critical points of their boundary set can be odd [12]. Here by critical points we mean points which are at the intersection of at least three $\partial D_{i}$ 's.

\subsection{Main goals}

In [4], we have combined results of [12] and [13] with efficient numerical computations to exhibit some candidates to be minimal 3-partitions for the square, the disk, .... This approach was based on an assumption of symmetry permitting the reduction to a more 
standard spectral analysis and consequently only gave symmetric candidates. Using two different symmetries of the square, we get the surprise of finding two candidates $\mathcal{D}_{1}$ and $\mathcal{D}_{2}$ with $\Lambda\left(\mathcal{D}_{1}\right)=\Lambda\left(\mathcal{D}_{2}\right)(\simeq 66.581)$ and give numerical evidence that the critical point for these partitions is at the center of the square. These candidates are represented in Figure 1. This leads naturally to questions of isospectrality which were solved using the
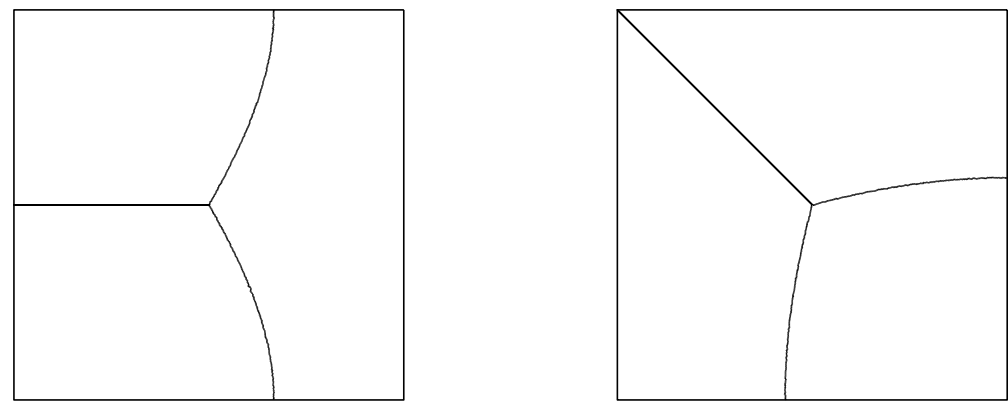

Figure 1: Two candidates with different symmetries for the minimal 3-partition of the square.

AB $X$-Hamiltonians in [3] with $X=C$, the center of the square. It was also sketched there an alternative proof using the construction of a double covering of the punctured square; this approach seems more adequate for performing the numerical analysis because of possible numerical difficulties in treating the singularities due to the Aharonov-Bohm potential and the fact that the eigenfunctions would have been complex-valued. This kind of arguments also appears in a similar context in [16] and [15].

We will push this numerical analysis with several goals:

- Illustrate the fact that these two symmetric candidates for minimal 3-partitions on the square belong actually to a continuous family of non necessarily symmetric candidates (see Figure 2 and Figure 9).

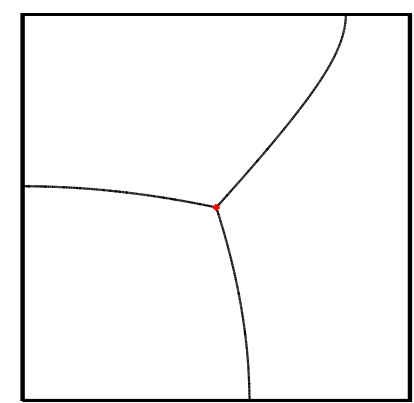

Figure 2: An asymmetric candidate for the minimal 3-partition of the square.

- Check, by moving the pole $X$ of the $\mathbf{A B} X$-Hamiltonian, the conjecture that the singular point of the minimal 3-partition is at the center.

- Understand and illustrate the mechanism of deformation of the nodal set, and hence extend or guess, in connection with recent papers of B. Noris and S. Terracini [18, 19], some of the properties described in Berger-Rubinstein [2] and Helffer-M.-and-T. Hoffmann-Ostenhof-Owen [10] for the ground state energy (see also [1]). 


\subsection{Organization of the paper}

In Section 2, we explain how we implement the computations on the double covering of the punctured square. In Section 3, we apply Courant's theory for comparing the AharonovBohm eigenvalues associated to this puncturing to the eigenvalues of the square.

Section 4 analyzes the dependence of the eigenfunctions of the Dirichlet Laplacian on the puncturing point appearing in the construction of the double covering.

Sections 5, 6 are more specifically devoted to the analysis of the behavior of the nodal sets and eigenvalues when the poles belong respectively to the perpendicular bisector $y=\frac{1}{2}$, the diagonal $y=x$, which correspond to cases when some symmetry of the square is respected in the puncturing. We treat also the case of the axis $y=\frac{1}{4}+\frac{x}{2}$ as an example of a generic situation.

Section 7 describes the possible applications of our analysis of nodal sets to the research of minimal partitions with a given topological type.

\section{Acknowledgements.}

The authors would like to thank Thomas Hoffmann-Ostenhof, Luc Hillairet and Susanna Terracini for useful discussions around this topics. We have been in particular stimulated by successive versions of [18]. Discussions about numerics with François Alouges and Grégory Vial were very fruitful. G. Vial helps us also by realizing numerous meshes for the computations and also for the detection of the nodal lines.

This paper has been partially written during the stay of the authors at the Erwin Schrödinger Institute from May to July 2009 and the authors are grateful for the very good working conditions and also for the fruitful discussions with other participants of the workshop on Topics in Spectral Theory.

\section{Numerical implementation}

The $\mathbf{A B} X$-Hamiltonian has a singularity at the pole $X$ and the eigenfunctions are complexvalued. For these reasons, we prefer to deal with the Dirichlet Laplacian on the doublecovering $\dot{\Omega}_{X}^{\mathcal{R}}$ whose eigenfunctions are real-valued. Some of these eigenfunctions, which will be described below, are directly related with the $K_{X}$-real eigenfunctions of the $\mathbf{A B} X$ Hamiltonian.

The numerical results were realized by using the Finite Element Library MÉLINA (see [17]). The computations consist only of the determination of the eigenfunctions of a Dirichlet Laplacian. Nevertheless, since we are interested in the nodal lines of these eigenfunctions, computations have to be quite accurate and we choose the package MéLinA permitting the implementation of high order elements. In our computations, we use for the approximation polynomials of degree 6 in each triangle of the discretization.

The main point of the numerical part consists in meshing the double covering $\dot{\Omega}_{X}^{\mathcal{R}}$ of the punctured domain $\Omega \backslash\{X\}$. To do this, we use the two-dimensional mesh generator TRIANGLE (see [20]). Let us explain in more details how we proceed.

Let $\Omega$ be the square $[0,1] \times[0,1]$ and $X$ be a point in $\left[0, \frac{1}{2}\right] \times\left[0, \frac{1}{2}\right]$. We start with meshing the $\Omega$ so that (see Fig. 3):

- the segment joining $(0,0)$ to the pole $X$ does not go through any element of the 
mesh,

- the segment $[(0,0) ; X]$ is the union of edges of an even number of triangles,

- the pole $X$ is the vertex of some triangles.

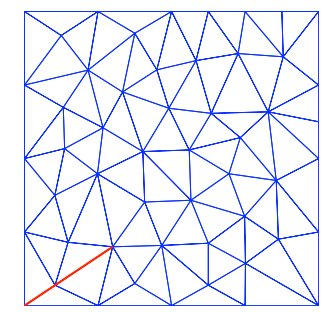

Figure 3: Mesh of the double covering $\dot{\Omega}_{X}^{\mathcal{R}}$.

This first mesh is essentially done for the first sheet and we repeat this mesh for the second sheet. To obtain a mesh of the double covering $\dot{\Omega}_{X}^{\mathcal{R}}$, we have to exchange the vertex along the segment $[(0,0) ; X]$ between the first and second sheet: we choose an orientation and we decide, for example to exchange the vertices between the first and second sheet of the triangles which are at the left of the segment. Then we remove the point $X$ of the second sheet by equaling it to the vertex $X$ of the first sheet.

In this paper $X$ is an element of the lattice of the first quarter of the square $\left[0, \frac{1}{2}\right] \times$ $\left[0, \frac{1}{2}\right]$ with step size $\frac{1}{100}$.

Theoretically, the eigenvalues and eigenfunctions depend only on the pole and are independent of the cut chosen for our construction. The introduction of the segment $[(0,0) ; X]$ is only a technical point and we have verified that the numerical computations of the eigenfunctions and eigenvalues are (with a rather good accuracy $\sim 10^{-3}$ ) independent of the choice of the line joining the pole to the boundary, that is the line between the first and second sheet. This is illustrated in Table 1 where we give the first fourteen eigenvalues of the Dirichlet Laplacian on the double covering of the square $\dot{\Omega}_{C}^{\mathcal{R}}$ realized with two different cutting lines: the half-diagonal and the half-perpendicular bisector.

Many computations are available on the web page (see [5]):

http://w3.bretagne.ens-cachan.fr/math/simulations/MinimalPartitions

The eigenvalues given all along this paper provide from our computations with an approximation at $5 \cdot 10^{-4}$ of the computed eigenvalues.

We observe that the eigenvalues of the square are numerically computed with a good accuracy whereas it is more difficult to be accurate for the $\mathbf{A B} X$-eigenvalues, particularly in the case of multiplicity 2 . In the following, we use a $\mathbb{P}^{6}$ approximation with at least 6000 elements.

To detect the nodal lines, we use a program realized by G. Vial. The idea is that it is very easy to compute the zero set of linear functions. In our case, we deal with a function which is piecewise $\mathbb{P}^{k}$ and given by a finite element method. We know the values of this function at some points. As soon as we have these values, we can replace this function by a new function which is piecewise linear. For this, we introduce some new points by an interpolation method. Then we detect the zero set of this new function. 


\begin{tabular}{|c|c|c|c|c|c|c|}
\hline \# elements & 3110 & 4410 & 6194 & 3102 & 4362 & 6254 \\
\hline 1 & 19.739 & 19.739 & 19.739 & 19.739 & 19.739 & 19.739 \\
\hline 2 & 33.518 & 33.509 & 33.510 & 33.517 & 33.511 & 33.510 \\
\hline 3 & 33.521 & 33.512 & 33.512 & 33.519 & 33.514 & 33.510 \\
\hline 4 & 49.348 & 49.348 & 49.348 & 49.348 & 49.348 & 49.348 \\
\hline 5 & 49.348 & 49.348 & 49.348 & 49.348 & 49.348 & 49.348 \\
\hline 6 & 66.581 & 66.581 & 66.581 & 66.581 & 66.581 & 66.581 \\
\hline 7 & 66.581 & 66.581 & 66.581 & 66.581 & 66.581 & 66.581 \\
\hline 8 & 78.957 & 78.957 & 78.957 & 78.957 & 78.957 & 78.957 \\
\hline 9 & 98.696 & 98.696 & 98.696 & 98.696 & 98.696 & 98.696 \\
\hline 10 & 98.696 & 98.696 & 98.696 & 98.696 & 98.696 & 98.696 \\
\hline 11 & 111.910 & 111.910 & 111.910 & 111.910 & 111.910 & 111.910 \\
\hline 12 & 111.910 & 111.910 & 111.910 & 111.910 & 111.910 & 111.910 \\
\hline 13 & 122.231 & 122.209 & 122.210 & 122.229 & 122.212 & 122.209 \\
\hline 14 & 122.240 & 122.215 & 122.217 & 122.235 & 122.220 & 122.211 \\
\hline
\end{tabular}

Table 1: Eigenvalues of the Dirichlet Laplacian on $\dot{\Omega}_{C}^{\mathcal{R}}$ for two meshes.

\section{A few theoretical comparison theorems}

\subsection{Notation}

We denote by $\Omega$ the square $[0,1] \times[0,1]$ and by $C=\left(\frac{1}{2}, \frac{1}{2}\right)$ the center of the square. We compute the eigenfunctions of the Laplacian on the double covering $\dot{\Omega}_{X}^{\mathcal{R}}$ of $\dot{\Omega}_{X}=\Omega \backslash\{X\}$. By a symmetry argument, it is enough to consider $X=\left(x_{0}, y_{0}\right)$ in the quarter square $\left[0, \frac{1}{2}\right] \times\left[0, \frac{1}{2}\right]$.

There are two ways of labelling the eigenvalues.

We can label it in the standard way and this is the way we get the eigenvalues in our numerical program. With this in mind, we denote by $\lambda_{k}\left(\dot{\Omega}_{X}^{\mathcal{R}}\right)$ the $k$-th eigenvalue of the Dirichlet Laplacian on $\dot{\Omega}_{X}^{\mathcal{R}}$.

We can also take account of the symmetry relative to the deck map $\mathcal{D}_{X}^{\mathcal{R}}$ associating to a given point in the covering the distinct point with same projection by the covering map $\pi_{X}^{\mathcal{R}}$ of $\dot{\Omega}_{X}^{\mathcal{R}}$ onto $\dot{\Omega}_{X}$. This splits the spectrum into two independent spectra relative to two orthogonal spaces in the $L^{2}$ space of our covering square.

The eigenvalues correspond

- either to eigenfunctions lifted from the eigenfunctions (of the Dirichlet Laplacian) ${ }^{1}$ on the square by the covering map (sometimes called $\mathcal{D}_{X}^{\mathcal{R}}$-symmetric because they are symmetric with respect to the deck map),

\footnotetext{
${ }^{1}$ We sometimes speak more shortly of spectrum of the square.
} 
- or to eigenfunctions which are $\mathcal{D}_{X}^{\mathcal{R}}$-antisymmetric with respect to the deck map.

We also call the second ones $\mathbf{A B} X$-eigenvalues because they can be seen as eigenvalues of an AB-Hamiltonian with a pole $X$ creating a (renormalized) flux equal to $\frac{1}{2}$. We shortly write $\mathbf{A B} X$-Hamiltonian, if we want to make explicit the reference to the pole. We denote by $\lambda_{k}^{\mathbf{A B} X}=\lambda_{k}^{\mathbf{A B}}\left(\dot{\Omega}_{X}\right)=\lambda_{k}^{A S}\left(\dot{\Omega}_{X}^{\mathcal{R}}\right)$ the $k$-th eigenvalue of the AB-Hamiltonian with pole at $X$. We denote by $\lambda_{k}^{S}\left(\dot{\Omega}_{X}^{\mathcal{R}}\right)=\lambda_{k}(\Omega)$ the $k$-th eigenvalue corresponding to the $\mathcal{D}_{X^{-}}^{\mathcal{R}}$ symmetric eigenvalues.

We will also observe that, when puncturing on the perpendicular bisectors or the diagonals of the square, additional symmetries will be important for explaining effective crossings of eigenvalues.

\subsection{Eigenvalues of the square}

The eigenvalues of the square are well known and given by the double sequence $\pi^{2}\left(m^{2}+n^{2}\right)$ with $m \in \mathbb{N} \backslash\{0\}, n \in \mathbb{N} \backslash\{0\}$, with corresponding basis of eigenfunctions given by

$$
\Omega \ni(x, y) \mapsto \phi_{m n}(x, y):=\sin (m \pi x) \sin (n \pi y) .
$$

Labelling the eigenvalues in increasing order leads to the sequence denoted by $\lambda_{k}(\Omega), k \in$ $\mathbb{N}^{*}$. Table 2 gives the first thirteen eigenvalues and the nodal set of the associated eigenfunctions belonging to the above basis. The second, fifth, seventh, ninth and twelfth eigenvalues are double and consequently, it is also natural to look at the nodal sets of linear combinations in order to determine all the possible nodal configurations associated with this eigenvalue.

Eigenvalues of the square

$$
\begin{aligned}
& \lambda_{1}(\Omega)=2 \pi^{2} \simeq 19.739 \\
& \lambda_{2}(\Omega)=\lambda_{3}(\Omega)=5 \pi^{2} \simeq 49.348 \\
& \lambda_{4}(\Omega)=8 \pi^{2} \simeq 78.957 \\
& \lambda_{5}(\Omega)=\lambda_{6}(\Omega)=10 \pi^{2} \simeq 98.696 \\
& \lambda_{7}(\Omega)=\lambda_{8}(\Omega)=13 \pi^{2} \simeq 128.305 \\
& \lambda_{9}(\Omega)=\lambda_{10}(\Omega)=17 \pi^{2} \simeq 167.783 \\
& \lambda_{11}(\Omega)=18 \pi^{2} \simeq 177.653 \\
& \lambda_{12}(\Omega)=\lambda_{13}(\Omega)=20 \pi^{2} \simeq 197.392
\end{aligned}
$$

$(m, n)$-labelling Nodal sets for $\phi_{m n}$
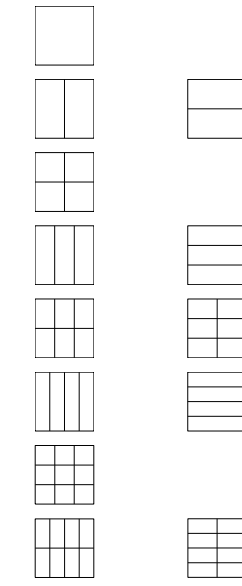

Table 2: First thirteen eigenvalues of the Dirichlet Laplacian on $\Omega$ and nodal set for the associated basis $\phi_{m n}$.

We notice that the $\mathcal{D}_{X}^{\mathcal{R}}$-symmetric spectrum of the Dirichlet Laplacian on the double covering $\dot{\Omega}_{X}^{\mathcal{R}}$ is the spectrum of the square and is independent of the pole. This is a consequence of the fact that the spectra of the Dirichlet Laplacian in $\Omega$ and $\dot{\Omega}_{X}$ are the same, the puncturing point being of capacity 0. So it is more the $\mathbf{A B} X$-spectrum which 
is of interest because depending on the position of the pole. Nevertheless, the standard labelling of all the eigenvalues on $\dot{\Omega}_{X}^{\mathcal{R}}$ can play a role when applying Courant's theorem. Of course we have $\lambda_{1}\left(\dot{\Omega}_{X}^{\mathcal{R}}\right)=\lambda_{1}(\Omega)$.

\subsection{Theoretical estimates of the eigenvalues}

This subsection is concerned with the comparison between the spectrum on the square, the spectrum on the double covering $\dot{\Omega}_{X}^{\mathcal{R}}$ and the $\mathbf{A B} X$-spectrum. We propose some equalities and upper-bounds between the eigenvalues essentially based on the minimax principle and on the Courant's nodal theorem which is recalled now.

\section{Theorem 3.1}

Let $k \geq 1, \lambda_{k}(\Omega)$ be the $k$-th eigenvalue for the Dirichlet Laplacian on $\Omega$. Then the number of nodal domains of any associated eigenfunction is bounded from above by $k$.

We would also apply this theorem for the $K_{X}$-real eigenfunctions of the $\mathbf{A B} X$-Hamiltonian on $\dot{\Omega}_{X}$. Equivalently, this corresponds to a Courant nodal theorem for the $\mathcal{D}_{X}^{\mathcal{R}}$-antisymmetric eigenfunctions on $\dot{\Omega}_{X}^{\mathcal{R}}$, already discussed in [3]. Combination of the Courant nodal theorem and Max-Min principle for the $\mathbf{A B} X$-Hamiltonian leads to the following proposition.

\section{Proposition 3.2}

Let $X \in\left[0, \frac{1}{2}\right] \times\left[0, \frac{1}{2}\right]$, then

$$
\lambda_{1}(\Omega)=\lambda_{1}\left(\dot{\Omega}_{X}^{\mathcal{R}}\right), \quad \lambda_{1}^{\mathbf{A B} X}=\lambda_{2}\left(\dot{\Omega}_{X}^{\mathcal{R}}\right), \quad \lambda_{2}^{\mathbf{A B} X}=\lambda_{3}\left(\dot{\Omega}_{X}^{\mathcal{R}}\right),
$$

and

$$
\begin{aligned}
& \lambda_{2}^{\mathbf{A B} X}<\quad \lambda_{2}(\Omega)=\lambda_{\ell_{2}}\left(\dot{\Omega}_{X}^{\mathcal{R}}\right) \text { with } \ell_{2} \geq 4 \text { (with multiplicity at least 2) }, \\
& \lambda_{3}^{\mathbf{A B} X} \leq \lambda_{4}(\Omega)=\lambda_{\ell_{4}}\left(\dot{\Omega}_{X}^{\mathcal{R}}\right) \text { with } \ell_{4} \geq 7, \\
& \lambda_{5}(\Omega)=\lambda_{\ell_{5}}\left(\dot{\Omega}_{X}^{\mathcal{R}}\right) \text { with } \ell_{5} \geq 8 \\
& \lambda_{5}^{\mathbf{A B} X} \leq \lambda_{7}(\Omega)=\lambda_{\ell_{7}}\left(\dot{\Omega}_{X}^{\mathcal{R}}\right) \text { with } \ell_{7} \geq 12 \\
& \lambda_{9}(\Omega)=\lambda_{\ell_{9}}\left(\dot{\Omega}_{X}^{\mathcal{R}}\right) \text { with } \ell_{9} \geq 14 \\
& \lambda_{8}^{\mathbf{A B} X} \leq \lambda_{11}(\Omega)=\lambda_{\ell_{11}}\left(\dot{\Omega}_{X}^{\mathcal{R}}\right) \text { with } \ell_{11} \geq 19 .
\end{aligned}
$$

If $X$ belongs to the perpendicular bisectors of the square, we have more accurately:

$$
\begin{aligned}
& \ell_{4}=\ell_{4}(X) \geq 8, \\
& \lambda_{6}^{\mathbf{A B} X} \leq \lambda_{7}(\Omega) .
\end{aligned}
$$

\section{Remark 3.3}

The multiplicity of $\lambda_{\ell}(\Omega)$ as eigenvalue of the Dirichlet Laplacian on $\dot{\Omega}_{X}^{\mathcal{R}}$ is of course larger or equal to its multiplicity on $\Omega$. This could permit to improve some inequalities above when we can find for a given pole an eigenfunction $u$ of the Dirichlet Laplacian on $\Omega$ vanishing at the pole. The number of nodal domains of the lifted symmetric function on the covering is then two times the number $\mu(u)$ of nodal domains of u instead of $2 \mu(u)-1$. To find this eigenfunction could be easier when the eigenspace is of higher dimension. This appears for example for $\lambda_{2}(\Omega)$. 
Proof : Let us first prove (3.2). We first observe that, for any $X \in\left[0, \frac{1}{2}\right] \times\left[0, \frac{1}{2}\right]$, there exists an eigenfunction $u_{X}$ of the Dirichlet Laplacian associated with $\lambda_{2}(\Omega)$ and $u_{X}(X)=$ 0 . We have just to look for $u_{X}$ of the form $u_{X}=\alpha \phi_{1,2}+\beta \phi_{2,1}$ with $(\alpha, \beta) \neq(0,0)$ satisfying $\alpha \phi_{1,2}(X)+\beta \phi_{2,1}(X)=0$. By lifting on $\dot{\Omega}_{X}^{\mathcal{R}}$, this gives a $\mathcal{D}_{X}^{\mathcal{R}}$-symmetric eigenfunction $u_{X} \circ \pi_{X}^{\mathcal{R}}$ on $\dot{\Omega}_{X}^{\mathcal{R}}$ with four nodal domains and associated with $\lambda_{2}(\Omega)$. Hence by Courant's theorem, $\lambda_{2}(\Omega)=\lambda_{\ell_{2}}\left(\dot{\Omega}_{X}^{\mathcal{R}}\right)$ with $\ell_{2} \geq 4$. To establish the first inequality in (3.2), we consider the functions $\max \left(u_{X}, 0\right)$ and $\max \left(-u_{X}, 0\right)$, which span a two-dimensional space in the form domain of the $\mathbf{A B} X$-Hamiltonian for which the energy is less than $\lambda_{2}(\Omega)$. We can conclude by the minimax principle. It is easy to see that the inequality is strict. Hence at this stage we also get (3.1).

Let us now prove (3.3). Using the three functions obtained by restriction to one nodal domain of the function $\phi_{2,2}$ (then extended by 0 ) which does not contain $X$, we obtain a 3-dimensional space of functions in the form domain of the $\mathbf{A B} X$-Hamiltonian for which the energy is less than $\lambda_{4}(\Omega)$ (or a 4-dimension space if $X$ is on the perpendicular bisector to the side of the square because we can in this case get a 4-dimensional space, see Remark 3.3). We then conclude by the minimax principle. The relation with $\lambda_{\ell_{4}}\left(\dot{\Omega}_{X}^{\mathcal{R}}\right)$ is an application of the Courant's nodal theorem using the function $\phi_{2,2} \circ \pi_{X}^{\mathcal{R}}$.

Relation (3.4) is a consequence of (3.3).

For (3.5), we can this time use the function $\phi_{3,2}$ which has at least 5 nodal domains not containing $X$. For $X$ on the perpendicular bisector, we get (3.9).

The function $\phi_{4,1}$ has at least 3 nodal domains not containing $X$. Using (3.5) and the multiplicity of $\lambda_{9}(\Omega)$, we obtain (3.6). (3.7).

Using the function $\phi_{3,3}$ which has at least 8 nodal domains not containing $X$, we deduce

The lower bound for $\ell_{7}, \ell_{9}$ and $\ell_{11}$ results immediately of the upper bounds of $\lambda_{5}^{\mathbf{A B} X}$ by $\lambda_{7}(\Omega)$ (hence by $\lambda_{9}(\Omega)$ ) and of $\lambda_{8}^{\mathbf{A B} X}$ by $\lambda_{11}(\Omega)$ established in (3.5) and (3.7).

\section{Lemma 3.4}

The nodal set of the second $K_{X}$-real eigenfunction $u_{2}^{\mathrm{AB} X}$ consists of one line joining the pole $X$ to the boundary.

\section{Proof :}

We know from [10] that a piecewise regular line in the nodal set should join the pole $\mathrm{X}$ to the boundary. Another piece in the nodal set should necessary create an additional nodal domain which will lead to $\lambda_{2} \leq \lambda_{2}^{\mathbf{A B} X}$ in contradiction to (3.2).

Let $X=(x, y) \in\left[0, \frac{1}{2}\right] \times\left[0, \frac{1}{2}\right]$, we denote $\tilde{X}=\left(\frac{x}{2}, \frac{y}{2}\right)$. Using a dilation argument, we can establish in complement of (3.3), that

$$
\lambda_{4}^{\mathbf{A B} X} \leq 4 \lambda_{1}^{\mathbf{A B} X} .
$$

The property that $\lambda_{1}^{\mathbf{A B X}} \geq \lambda_{1}(\Omega)$ is of course a particular case of the diamagnetic inequality. We will observe on the pictures that the situation is much more complicate for the excited states. Except in the case of additional symmetries where some monotonicity will be proven, we have no theoretical results. 


\section{Remark 3.5}

We will see in Figures 18, 22 and 26, that the upper-bounds (3.2), (3.3) and (3.5) in Proposition 3.2 for the $\mathbf{A B} X$-eigenvalues are optimal in the sense that we can find a pole such that the upper-bound is false with a smaller eigenvalue of the square.

\section{Behavior of the eigenvalues on the double covering of the punctured square, when moving the pole}

In this section, we start to discuss the influence of the location of the puncturing point $X$ (or pole) on the topological structure of the nodal set of the first eigenfunctions.

\subsection{Behavior when the pole tends to the boundary}

It has been announced by B. Noris and S. Terracini $[18,19]$ that the $k$-th $\mathbf{A B} X$-eigenvalue of the punctured square tends to the $k$-th eigenvalue of the Dirichlet Laplacian on the square as the pole tends to the boundary (see also [14] for connected results). They also establish in [18] the continuity with respect to a pole and prove that $X \mapsto \lambda_{k}^{\mathbf{A B} X}$ is of class $C^{1}$ if $\lambda_{k}^{\mathrm{AB} X}$ is simple. Because after a translation by $X$, we get a fixed operator with moving regular boundary and fixed pole at $(0,0)$, the regularity is actually easy. These results are illustrated in Figures $4-14$ which represent the eigenvalues $\lambda_{k}\left(\dot{\Omega}_{X}^{\mathcal{R}}\right)$, $k=2,3,6,7,8,9,11,12,13$, according to the location of the pole $X \in \mathcal{P}$ and Table 3 which gives the first 12 eigenvalues of the Dirichlet Laplacian on $\dot{\Omega}_{X}^{\mathcal{R}}$ for three points $X$ : one near the boundary denoted by $A$, one at the center denoted by $C$ and one other denoted by $B$.

\begin{tabular}{|c|c|c|c|}
\hline$n$ & $\lambda_{n}\left(\dot{\Omega}_{A}^{\mathcal{R}}\right)$ & $\lambda_{n}\left(\dot{\Omega}_{B}^{\mathcal{R}}\right)$ & $\lambda_{n}\left(\dot{\Omega}_{C}^{\mathcal{R}}\right)$ \\
\hline 1 & 19.739 & 19.739 & 19.739 \\
2 & 19.739 & 20.269 & 33.528 \\
3 & 49.348 & 49.325 & 33.534 \\
4 & 49.348 & 49.348 & 49.348 \\
5 & 49.348 & 49.348 & 49.348 \\
6 & 49.348 & 51.480 & 66.581 \\
7 & 78.957 & 78.957 & 66.581 \\
8 & 78.957 & 79.536 & 78.957 \\
9 & 98.696 & 98.658 & 98.696 \\
10 & 98.696 & 98.696 & 98.696 \\
11 & 98.696 & 98.696 & 111.910 \\
12 & 98.696 & 102.647 & 111.910 \\
\hline
\end{tabular}

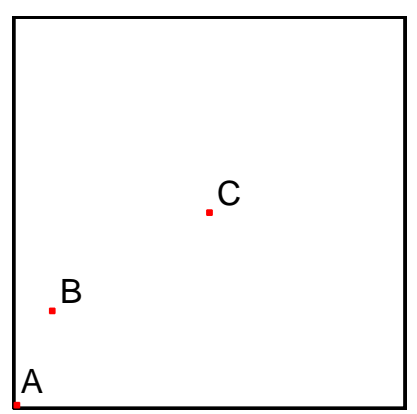

Table 3: First 12 eigenvalues of the Dirichlet Laplacian on $\dot{\Omega}_{A}^{\mathcal{R}}$, $\dot{\Omega}_{B}^{\mathcal{R}}$ and $\dot{\Omega}_{C}^{\mathcal{R}}$, with $A=\left(\frac{1}{100}, \frac{1}{100}\right), B=\left(\frac{1}{10}, \frac{2}{5}\right), C=\left(\frac{1}{2}, \frac{1}{2}\right)$. 


\subsection{Eigenvalues 2 and 3}

We observe numerically, see Figure 4 , that for $X \in \mathcal{P}$, the function $X \mapsto \lambda_{2}\left(\dot{\Omega}_{X}^{\mathcal{R}}\right)$ has a global maximum, denoted by $\lambda_{2}^{\max }$ for $X=C$ and is minimal when $X$ belongs to the boundary $x=0$ or $y=0$. This minimum equals $\lambda_{2}(\Omega)$. Moreover we do not observe other critical points in $\mathcal{P}$. Looking at Figure 5 , we observe numerically that the function $X \mapsto \lambda_{3}\left(\dot{\Omega}_{X}^{\mathcal{R}}\right)$ behaves conversely : it has a global minimum, denoted by $\lambda_{3}^{\min }$, for $X=C$ and the maximum is reached at the boundary $x=0$ or $y=0$ and equals $\lambda_{3}(\Omega)$. We have monotonicity along lines joining a point of the boundary to the center $C$. Furthermore, we notice that $\lambda_{2}^{\max }=\lambda_{3}^{\min }$.
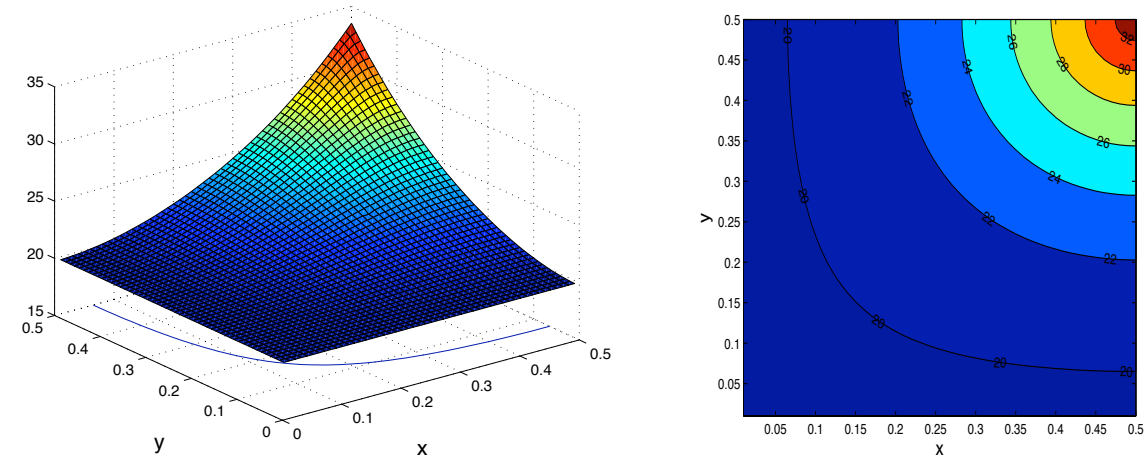

Figure 4: $X \mapsto \lambda_{2}\left(\dot{\Omega}_{X}^{\mathcal{R}}\right)$ for $X \in \mathcal{P}$.
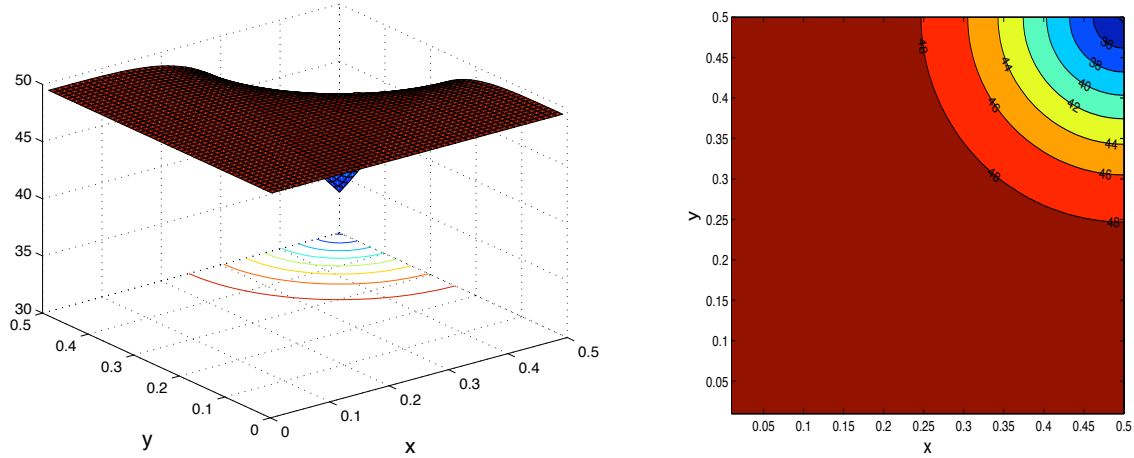

Figure 5: $X \mapsto \lambda_{3}\left(\dot{\Omega}_{X}^{\mathcal{R}}\right)$ for $X \in \mathcal{P}$.

Figure 6 gives the eigenvalues and the nodal lines of the eigenfunctions associated with the second and third eigenvalues of the Dirichlet Laplacian on $\dot{\Omega}_{X}^{\mathcal{R}}$ on the first and second lines respectively. The $j$-th column corresponds to the domain $\dot{\Omega}_{X_{j}}^{\mathcal{R}}$ with $X_{j}=\left(\frac{1}{5}, \frac{j}{10}\right)$, $j=1, \ldots, 5$. These figures are illustration of the theory of Berger-Rubinstein [2] and Helffer-Hoffmann-Ostenhof-Hoffmann-Ostenhof-Owen [10] (see also [1]). For the ground state energy, we recover the theorem of these authors that the nodal set is composed of a line joining the pole to the boundary. We observe that the nodal line in the first case is choosing a kind of minimal distance between the pole and the boundary whereas the nodal line in the second case seems to choose a kind of maximal distance. We do not have a rigorous explanation for this property except that it should be related to the theorem proved in $[2,10]$ that $\lambda_{1}^{\mathbf{A B X}}$ is the infimum over the Dirichlet eigenvalue of the Laplacian in $\Omega \backslash \gamma$ where $\gamma$ is a regular path joining the pole $X$ to the boundary. 


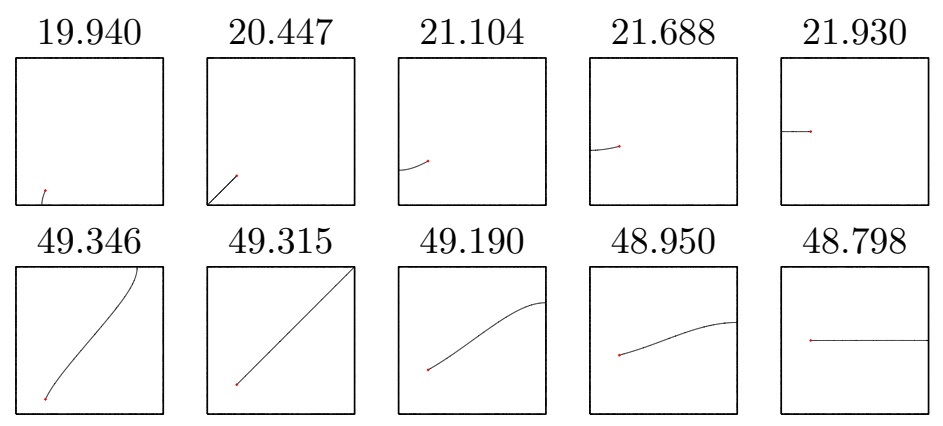

Figure 6: Nodal set for the eigenfunctions associated with $\lambda_{k}\left(\dot{\Omega}_{X}^{\mathcal{R}}\right), k=2,3$, for poles $X=\left(\frac{1}{5}, \frac{j}{10}\right), 1 \leq j \leq 5$.

We also recover the two last equations in (3.1).

\subsection{Eigenvalues 4 and 5}

Using (3.2), we have proved that $\lambda_{2}(\Omega) \geq \lambda_{5}\left(\dot{\Omega}_{X}^{\mathcal{R}}\right)$. We observe numerically (see also ahead Figures 18 and 22 for poles along a symmetry axis and Figure 26) that, for any $X \in \mathcal{P}$, we have

$$
\lambda_{4}\left(\dot{\Omega}_{X}^{\mathcal{R}}\right)=\lambda_{5}\left(\dot{\Omega}_{X}^{\mathcal{R}}\right)=\lambda_{2}(\Omega) .
$$

\subsection{Eigenvalues 6 and 7}
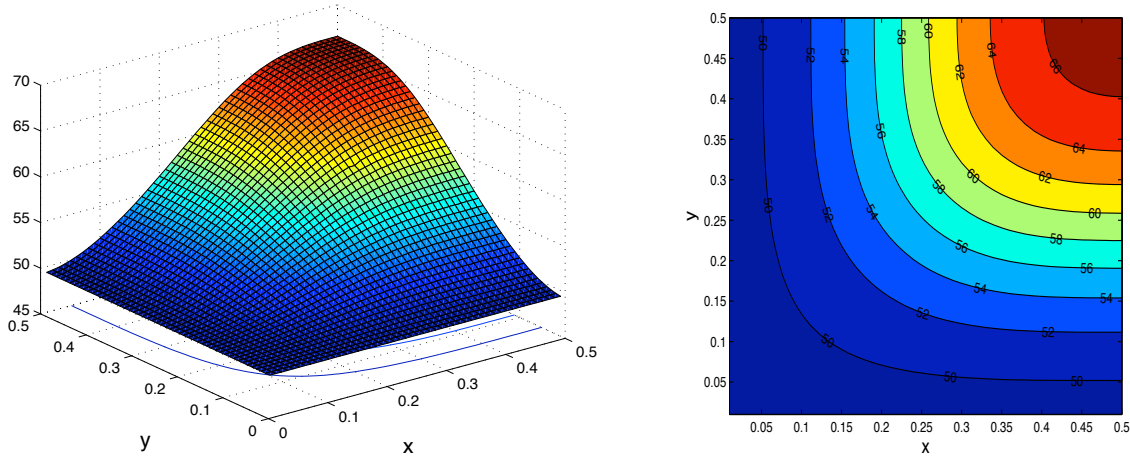

Figure 7: $\lambda_{6}\left(\dot{\Omega}_{X}^{\mathcal{R}}\right)$, in function of the pole $X \in \mathcal{P}$.

Numerics shows that the 6 -th eigenvalue $\lambda_{6}\left(\dot{\Omega}_{X}^{\mathcal{R}}\right)$ has a unique maximum $\lambda_{6}^{\max } \simeq 66.581$ which is attained when the pole is at the center and is minimal at the boundary. We do not observe other critical points. The 7-th eigenvalue $\lambda_{7}\left(\dot{\Omega}_{X}^{\mathcal{R}}\right)$ is minimal when the pole is at the center and its minimum $\lambda_{7}^{\min }$ is equal to $\lambda_{6}^{\max }$. When the pole is at the center, the zero set of the 6 -th eigenfunction provides, by projection, a candidate for a 3 -partition and $\lambda_{6}^{\max }$ is the conjectured value for $\mathfrak{L}_{3}(\Omega)$. We observe that the 7 -th eigenvalue becomes constant equal to $\lambda_{7}^{\max }=\lambda_{4}(\Omega)=8 \pi^{2}$ as a function of the pole when the pole is close to the boundary. Looking at the nodal set for the eigenfunctions associated with $\lambda_{7}\left(\dot{\Omega}_{X}^{\mathcal{R}}\right)$, we notice that when $X$ is close to the boundary, $\lambda_{7}\left(\dot{\Omega}_{X}^{\mathcal{R}}\right)=\lambda_{4}(\Omega)$. This corresponds to 

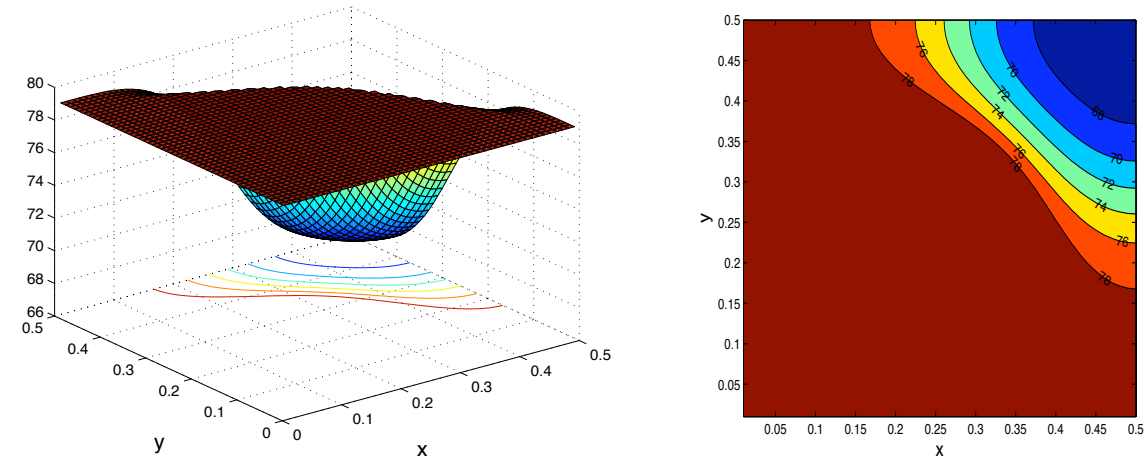

Figure 8: $\lambda_{7}\left(\dot{\Omega}_{X}^{\mathcal{R}}\right)$, in function of the pole $X \in \mathcal{P}$.

a crossing when $X$ approaches the boundary between fixed eigenvalues belonging to the spectrum of the square (i.e. the $\mathcal{D}_{X}^{\mathcal{R}}$-symmetric spectrum on the covering) and the $X$ dependent $\mathbf{A B} X$-spectrum (i.e. the $\mathcal{D}_{X}^{\mathcal{R}}$-antisymmetric spectrum on the covering $\dot{\Omega}_{X}^{\mathcal{R}}$ ).

Applying relation (3.3), we have proved theoretically that $\lambda_{4}(\Omega) \geq \lambda_{7}\left(\dot{\Omega}_{X}^{\mathcal{R}}\right)$. We observe numerically that this relation is optimal in the sense that we have equality for $X$ close to the boundary.

Considering the linear combination of the eigenfunctions $u_{6}$ and $u_{7}$ associated respectively with $\lambda_{6}\left(\dot{\Omega}_{C}^{\mathcal{R}}\right)$ and $\lambda_{7}\left(\dot{\Omega}_{C}^{\mathcal{R}}\right)$, with $C=\left(\frac{1}{2}, \frac{1}{2}\right)$, we can construct a family of 3-partitions with the same energy. Figures 9 give the projection by $\pi_{C}^{\mathcal{R}}$ of the nodal set for the functions $t u_{6}+(1-t) u_{7}$ with $t=k / 8, k=0, \ldots, 8$.
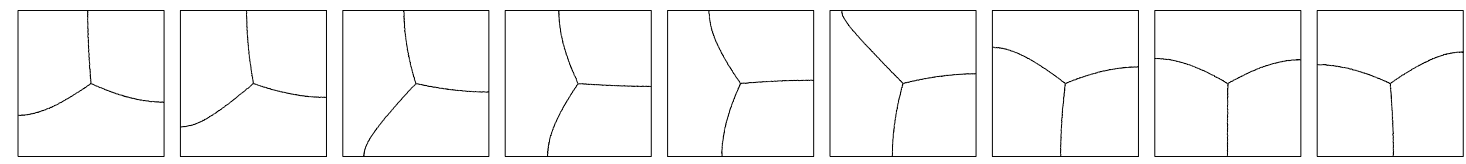

Figure 9: Continuous family of 3-partitions with the same energy.

Using the double covering approach, it is very easy, for a given eigenvalue with multiplicity $\geq 2$, to compute linear combinations of the two eigenfunctions given by the program, because their approximations are given at the same points. If we wanted to construct a family of 3-partitions with the same energy without this approach, we should, for example, construct linear combinations of the two candidates presented in Figure 1. Unfortunately the approximation of the eigenfunctions are not defined at the same points and we should defined new approximations at some new common points. This approach is more technical and requires a longer computation time than the double covering approach.

It is interesting to discuss if we can prove the numerically observed inequality

$$
\lambda_{3}^{\mathbf{A B} X} \geq \lambda_{2}(\Omega) .
$$

This is directly related to the conjecture proposed by S. Terracini ${ }^{2}$ :

\section{Conjecture 4.1}

Except at the center $X=C=\left(\frac{1}{2}, \frac{1}{2}\right), \lambda_{3}^{\mathbf{A B} X}$ is simple and the corresponding nodal set of

${ }^{2}$ Personal communication 
the $K_{X}$-real eigenfunction is the union of a line joining the pole to the boundary and of another line joining two points of the boundary.

We note indeed that if the conjecture is true, we will get (4.2) by the minimax principle. This conjecture is illustrated in Fig. A1, having in mind that $\lambda_{6}\left(\dot{\Omega}_{X}^{\mathcal{R}}\right)=\lambda_{3}^{\mathbf{A B} X}$.

\subsection{Eigenvalues 8 and 9}

Figures 10-11 represent the numerical computations of $\lambda_{8}\left(\dot{\Omega}_{X}^{\mathcal{R}}\right)$ and $\lambda_{9}\left(\dot{\Omega}_{X}^{\mathcal{R}}\right)$ for $X \in \mathcal{P}$. We observe numerically that the function $X \mapsto \lambda_{8}\left(\dot{\Omega}_{X}^{\mathcal{R}}\right)$ has a unique maximum denoted by $\lambda_{8}^{\max }$ at a point $C_{1}$ on the diagonal and $X \mapsto \lambda_{9}\left(\dot{\Omega}_{X}^{\mathcal{R}}\right)$ reaches its unique minimum, $\lambda_{9}^{\text {min }}$, at this point. We can recover this behavior on Figure 18 where are drawn the eigenvalues for poles on the diagonal. Numerically, $\lambda_{8}^{\max }=\lambda_{9}^{\min }$ and we come back to this equality in Subsection 6.3 where we look at the nodal lines of the eigenfunctions associated with $\lambda_{8}\left(\dot{\Omega}_{X}^{\mathcal{R}}\right)$ and $\lambda_{9}\left(\dot{\Omega}_{X}^{\mathcal{R}}\right)$ and predict the existence of the point $C_{1}$, see Figure 23.
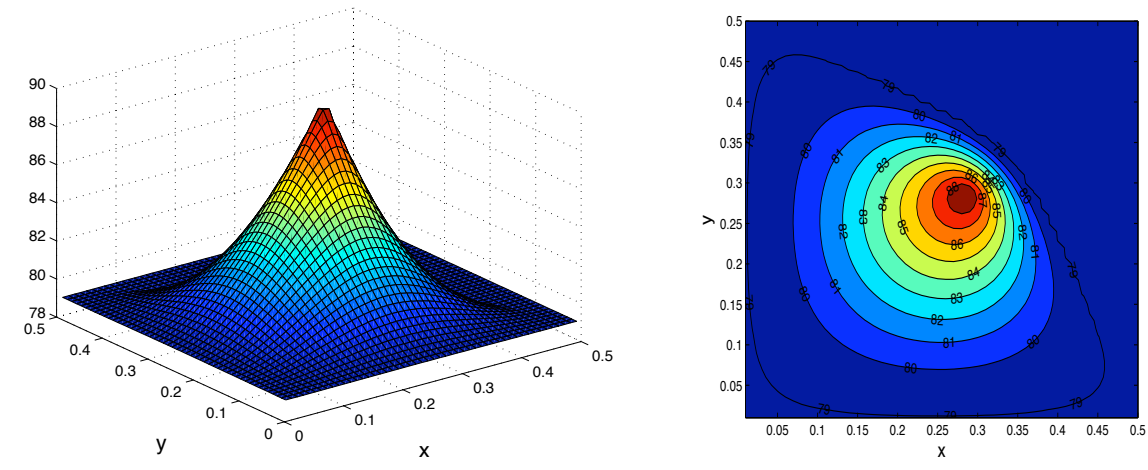

Figure 10: $X \mapsto \lambda_{8}\left(\dot{\Omega}_{X}^{\mathcal{R}}\right)$ for $X \in \mathcal{P}$.
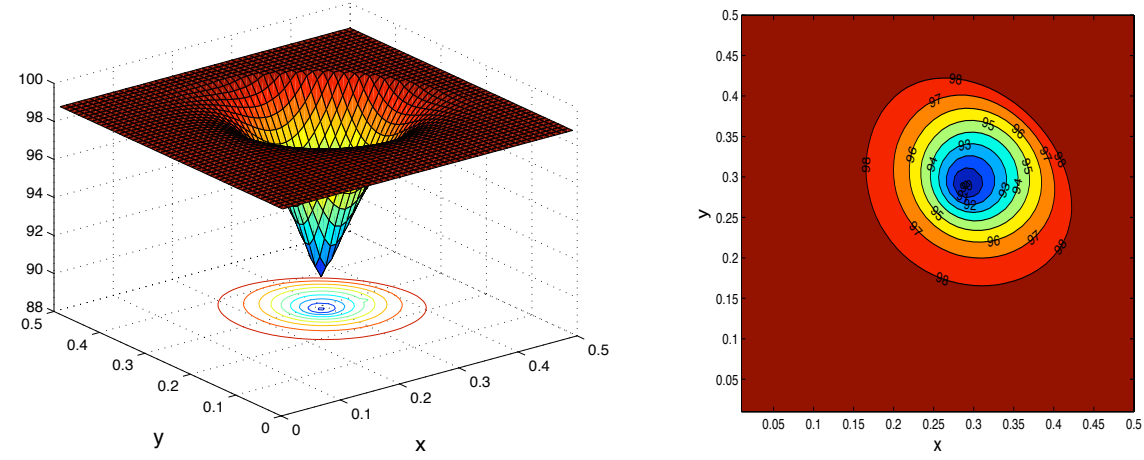

Figure 11: $X \mapsto \lambda_{9}\left(\dot{\Omega}_{X}^{\mathcal{R}}\right)$ for $X \in \mathcal{P}$.

According to (3.8), we have proved that $\lambda_{8}\left(\dot{\Omega}_{X}^{\mathcal{R}}\right) \leq \lambda_{7}(\Omega)$. This theoretical upperbound is coarse and the numerics suggests that we have in fact the better bound $\lambda_{8}\left(\dot{\Omega}_{X}^{\mathcal{R}}\right) \leq$ $\lambda_{5}(\Omega)$. Moreover $C_{1}$ is singular for the maps $X \mapsto \lambda_{8}\left(\dot{\Omega}_{X}^{\mathcal{R}}\right)$ and $\lambda_{9}\left(\dot{\Omega}_{X}^{\mathcal{R}}\right)$. 


\subsection{Eigenvalue 10}

We observe numerically that, for any $X \in \mathcal{P}$, we have

$$
\lambda_{10}\left(\dot{\Omega}_{X}^{\mathcal{R}}\right)=\lambda_{5}(\Omega) .
$$

What we have proven in (3.4) is weaker.

\subsection{Eigenvalues 11 and 12}
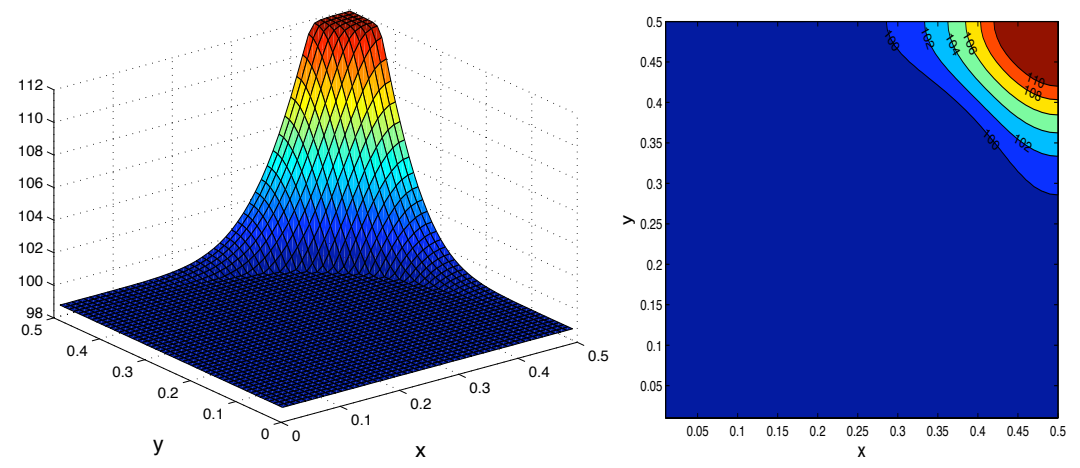

Figure 12: $\lambda_{11}\left(\dot{\Omega}_{X}^{\mathcal{R}}\right)$, according to the location of the pole $X \in \mathcal{P}$.
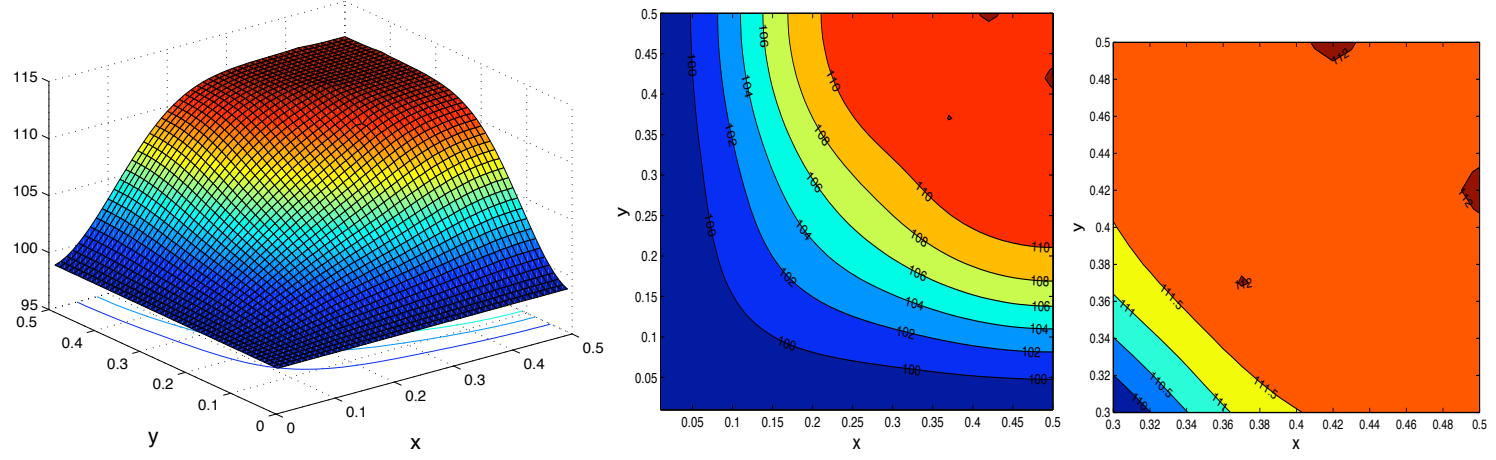

Figure 13: $\lambda_{12}\left(\dot{\Omega}_{X}^{\mathcal{R}}\right)$ according to $X \in \mathcal{P}$ with additional zoom.

As for the 7-th eigenvalue $\lambda_{7}\left(\dot{\Omega}_{X}^{\mathcal{R}}\right)$, the 11-th eigenvalue $\lambda_{11}\left(\dot{\Omega}_{X}^{\mathcal{R}}\right)$ becomes constant as $X$ is far enough from the center and equal to $\lambda_{5}(\Omega)$. It seems that $\lambda_{11}\left(\dot{\Omega}_{X}^{\mathcal{R}}\right)$ has a unique global maximum when the pole is at the center (see Figure 12). On the contrary, see Figure 13, it seems that $\lambda_{12}\left(\dot{\Omega}_{X}^{\mathcal{R}}\right)$ has only a local minimum at the center and admits local maxima at the points $C_{2}$ on the diagonal (see Figure 18) and $A_{1}=\left(a_{1}, \frac{1}{2}\right)$ and $A_{1}^{\prime}=\left(\frac{1}{2}, a_{1}\right)$ on the perpendicular bisector (see Figure 22). These local maxima can also be observed in Figures 19-24 where is given the 12-th eigenvalue around the points $A_{1}$ and $C_{2}$. With a finer grid for $X$ around $A_{1}=\left(a_{1}, \frac{1}{2}\right)$ and $C_{2}=\left(c_{2}, c_{2}\right)$, we get, thanks to the change of symmetry in the nodal domains, the estimates:

$$
\begin{aligned}
& 0.4255 \leq a_{1} \leq 0.4256 \quad \text { and } \quad 112.05 \leq \lambda_{12}\left(\dot{\Omega}_{A_{1}}^{\mathcal{R}}\right) \leq 112.06 \\
& 0.3689 \leq c_{2} \leq 0.3690 \quad \text { and } \quad 112.01 \leq \lambda_{12}\left(\dot{\Omega}_{C_{2}}^{\mathcal{R}}\right) \leq 112.02
\end{aligned}
$$


It seems that $X \mapsto \lambda_{12}\left(\dot{\Omega}_{X}^{\mathcal{R}}\right)$ presents a global maximum for $X=C_{2}$ and local maxima at $X=A_{2}=\left(a_{2}, \frac{1}{2}\right)$ and $X=\left(\frac{1}{2}, a_{2}\right)$ but the accuracy of our computation, although we used a refined grid for $X$, does not allow us to be more affirmative.

Theoretically, relation (3.5) gives $\lambda_{11}\left(\dot{\Omega}_{X}^{\mathcal{R}}\right) \leq \lambda_{7}(\Omega)$. This estimate is optimal since there exist poles for which $\lambda_{11}\left(\dot{\Omega}_{X}^{\mathcal{R}}\right)>\lambda_{5}(\Omega)=\lambda_{6}(\Omega)$ (see also Figures 18-22 for poles along the diagonal or the perpendicular bisector). We recall that $\lambda_{12}\left(\dot{\Omega}_{X}^{\mathcal{R}}\right)=\lambda_{6}^{\mathbf{A B}, X}$.

\subsection{Eigenvalues 13, 14 and 15}
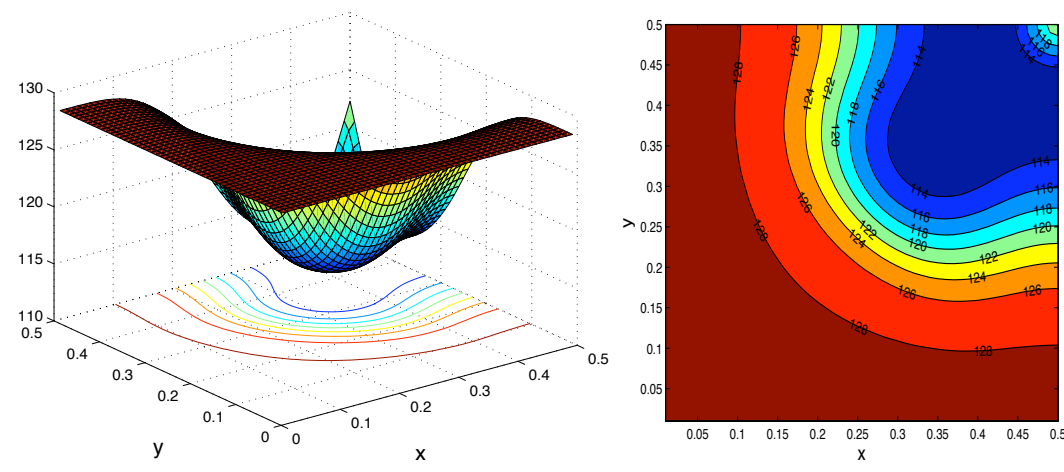

Figure 14: $\lambda_{13}\left(\dot{\Omega}_{X}^{\mathcal{R}}\right)$ according to $X \in \mathcal{P}$.
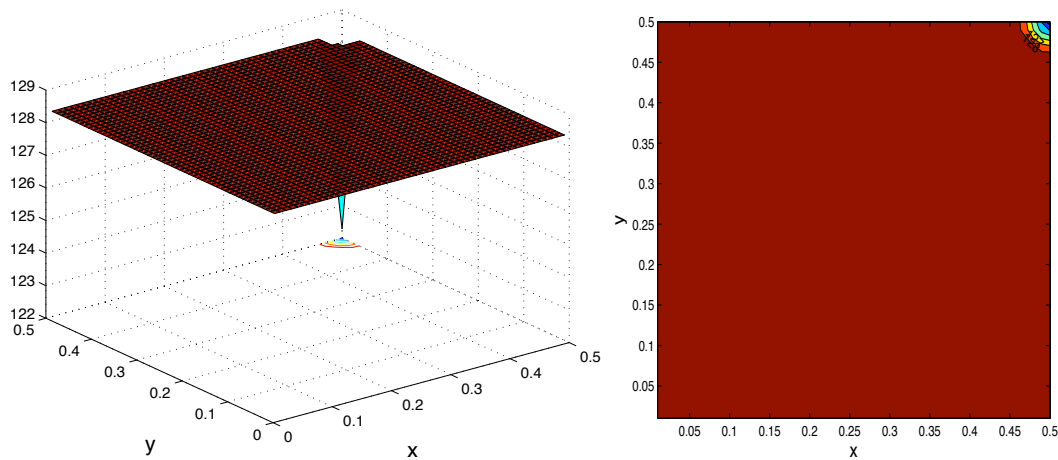

Figure 15: $\lambda_{14}\left(\dot{\Omega}_{X}^{\mathcal{R}}\right)$ according to $X \in \mathcal{P}$.

We observe that $X \mapsto \lambda_{13}\left(\dot{\Omega}_{X}^{\mathcal{R}}\right)$ tends to $\lambda_{7}(\Omega)$ when $X$ tends to the boundary and admits a local minimum for $X=C_{2}$ along the diagonal. It seems also that this function admits a local minimum for $X$ on one perpendicular bisector at points $A_{1}$ and $A_{1}^{\prime}$ (see Figures 18 and 22) and a local maximum, $\lambda_{13}^{C}$ at $X=C$.

Looking at Figure 15, we observe numerically that for $X$ sufficiently far from $C$, we have

$$
\lambda_{14}\left(\dot{\Omega}_{X}^{\mathcal{R}}\right)=\lambda_{7}(\Omega) .
$$

It seems that the function $X \mapsto \lambda_{14}\left(\dot{\Omega}_{X}^{\mathcal{R}}\right)$ reaches its unique minimum $\lambda_{14}^{\text {min }}$ at $X=C$ and we have $\lambda_{14}^{\min }=\lambda_{13}^{C}$, as observed in Figure 22 .

We observe numerically that, for any $X \in \mathcal{P}$, we have

$$
\lambda_{15}\left(\dot{\Omega}_{X}^{\mathcal{R}}\right)=\lambda_{7}(\Omega) .
$$




\subsection{Eigenvalues 16 and 17}

Looking at Figure 16, we observe that $X \mapsto \lambda_{16}\left(\dot{\Omega}_{X}^{\mathcal{R}}\right)$ admits three local maxima : two on the perpendicular bisectors reaching at points $A_{2}$ and $A_{2}^{\prime}$ and one on the diagonal at point $C_{3}$, see Figures 18 and 22. We also observe that $\lambda_{16}\left(\dot{\Omega}_{X}^{\mathcal{R}}\right)$ tends to $\lambda_{7}(\Omega)$ as $X$ tends to the boundary or $X$ tends to the center $X=C$.

Let us now deal with $\lambda_{17}\left(\dot{\Omega}_{X}^{\mathcal{R}}\right)$ represented in Figure 17. The behavior is in reverse order: the function $X \mapsto \lambda_{17}\left(\dot{\Omega}_{X}^{\mathcal{R}}\right)$ seems to have three local minima at the same points $A_{2}, A_{2}^{\prime}$ and $C_{3}$ and these minima equal the maxima of $\lambda_{16}\left(\dot{\Omega}_{X}^{\mathcal{R}}\right)$. With finer discretization around the points $A_{2}=\left(a_{2}, \frac{1}{2}\right)$ and $C_{3}=\left(c_{3}, c_{3}\right)$, we estimate numerically according to the change of symmetry in the nodal domains:

$$
\begin{aligned}
& 0.2389 \leq c_{3} \leq 0.2390 \quad \text { and } \quad 151.40 \leq \lambda_{16}\left(\dot{\Omega}_{C_{3}}^{\mathcal{R}}\right) \leq 151.42, \\
& 0.2829 \leq a_{2} \leq 0.2830 \quad \text { and } \quad 150.03 \leq \lambda_{16}\left(\dot{\Omega}_{A_{2}}^{\mathcal{R}}\right) \leq 150.05
\end{aligned}
$$

Consequently the extrema seem to be global at $C_{3}$ and local at $A_{2}$ and $A_{2}^{\prime}$. Furthermore, $\lambda_{16}\left(\dot{\Omega}_{X}^{\mathcal{R}}\right)$ tends to $\lambda_{9}(\Omega)$ as $X$ tends to the boundary or to the center $C$.
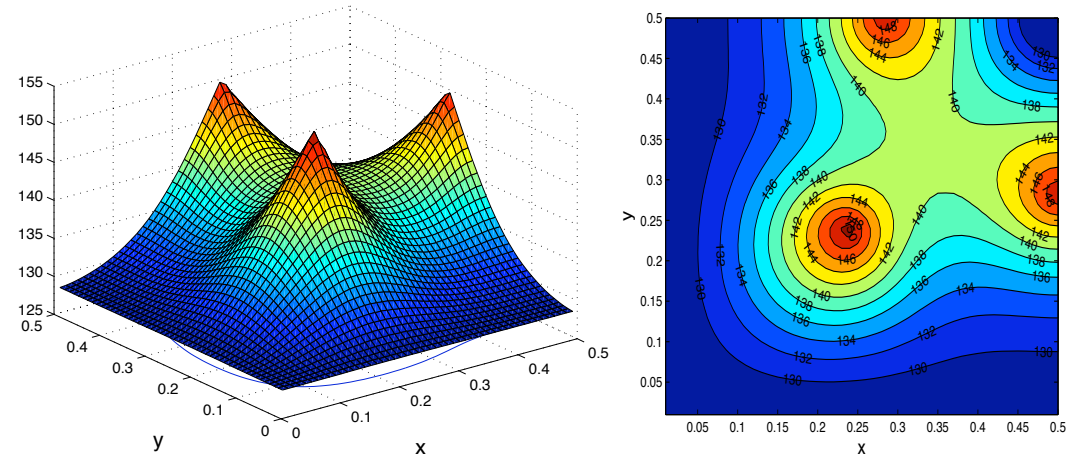

Figure 16: $\lambda_{16}\left(\dot{\Omega}_{X}^{\mathcal{R}}\right)$ according to $X \in \mathcal{P}$.
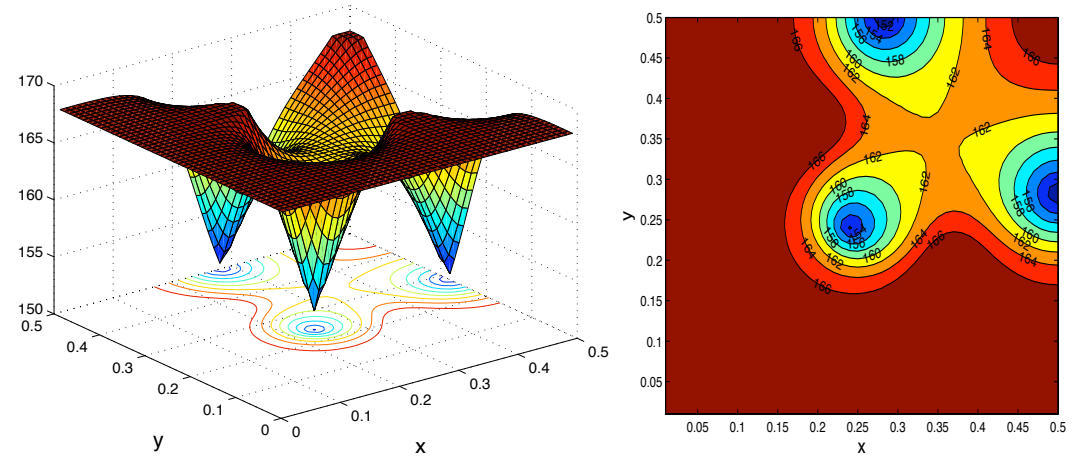

Figure 17: $\lambda_{17}\left(\dot{\Omega}_{X}^{\mathcal{R}}\right)$ according to $X \in \mathcal{P}$. 


\section{Moving the pole along the axis $y=1 / 2$}

\subsection{Analysis of the symmetries}

Let us begin with some considerations about the $\mathbf{A B} X$-Hamiltonian on the $X$-punctured square, using the symmetry along the perpendicular bisector $y=\frac{1}{2}$. We refer to [3] for more details. We fix a point $X=\left(x_{0}, \frac{1}{2}\right)$ along this line. The square is invariant under the symmetry

$$
\sigma_{1}(x, y)=(x, 1-y) .
$$

We consider the antilinear operator

$$
\Sigma_{1}^{c}=\Gamma \Sigma_{1},
$$

where $\Gamma$ is the complex conjugation $(\Gamma u=\bar{u})$ and $\Sigma_{1}$ is associated with $\sigma_{1}$ by the relation $\Sigma_{1} u(x, y)=u(x, 1-y)$.

We use the symmetry of the $X$-punctured square to give an orthogonal decomposition of $L_{K}^{2}=L_{K_{X}}^{2}$ :

$$
L_{K}^{2}=L_{K, \Sigma_{1}}^{2} \oplus L_{K, a \Sigma_{1}}^{2}
$$

where

$$
L_{K, \Sigma_{1}}^{2}=\left\{u \in L_{K}^{2}, \Sigma_{1}^{c} u=u\right\},
$$

and

$$
L_{K, a \Sigma_{1}}^{2}=\left\{u \in L_{K}^{2}, \Sigma_{1}^{c} u=-u\right\} .
$$

As established in [3, Lemma 5.6], we can prove that:

- if $u \in C^{\infty}\left(\dot{\Omega}_{X}\right) \cap L_{K, \Sigma_{1}}^{2}$, then its nodal set contains $\left[0, x_{0}\right] \times\left\{\frac{1}{2}\right\}$,

- if $u \in C^{\infty}\left(\dot{\Omega}_{X}\right) \cap L_{K, a \Sigma_{1}}^{2}$, then its nodal set contains $\left[x_{0}, 1\right] \times\left\{\frac{1}{2}\right\}$.

Dealing with a mixed Dirichlet-Neumann condition on the half-domain, we deduce that the eigenvalues for which the eigenfunctions are symmetric are increasing with respect to $x_{0}$, whereas the eigenvalues for which the eigenfunctions are antisymmetric are decreasing with respect to $x_{0}$.

\subsection{Spectral variation}

Figure 18 gives the eigenvalues for poles along the axis $y=1 / 2$ and $0<x \leq 1 / 2$. We observe that $\lambda_{k}^{\mathbf{A B} X}$ is increasing with the first coordinate of $X$ for $k=1,3,5,6$, whereas it is decreasing for $k=2,4$.

We denote by $X(x)=\left(x, \frac{1}{2}\right)$ the pole and introduce $A_{j}=X\left(a_{j}\right), B_{j}=X\left(b_{j}\right)$ specific points which can be seen on the Figure 18. The below mentioned symmetry (resp. antisymmetry) is in this section with respect to $\Sigma_{1}^{c}$ (see (5.2) and (5.3)) and denoted by $\Sigma_{1}$ (resp. a $\Sigma_{1}$ ) on the figure. Then, we observe numerically:

(a) $x \mapsto \lambda_{1}\left(\dot{\Omega}_{X(x)}^{\mathcal{R}}\right)$ equals $\lambda_{1}(\Omega)$, in adequation with the theoretical result (3.1). 


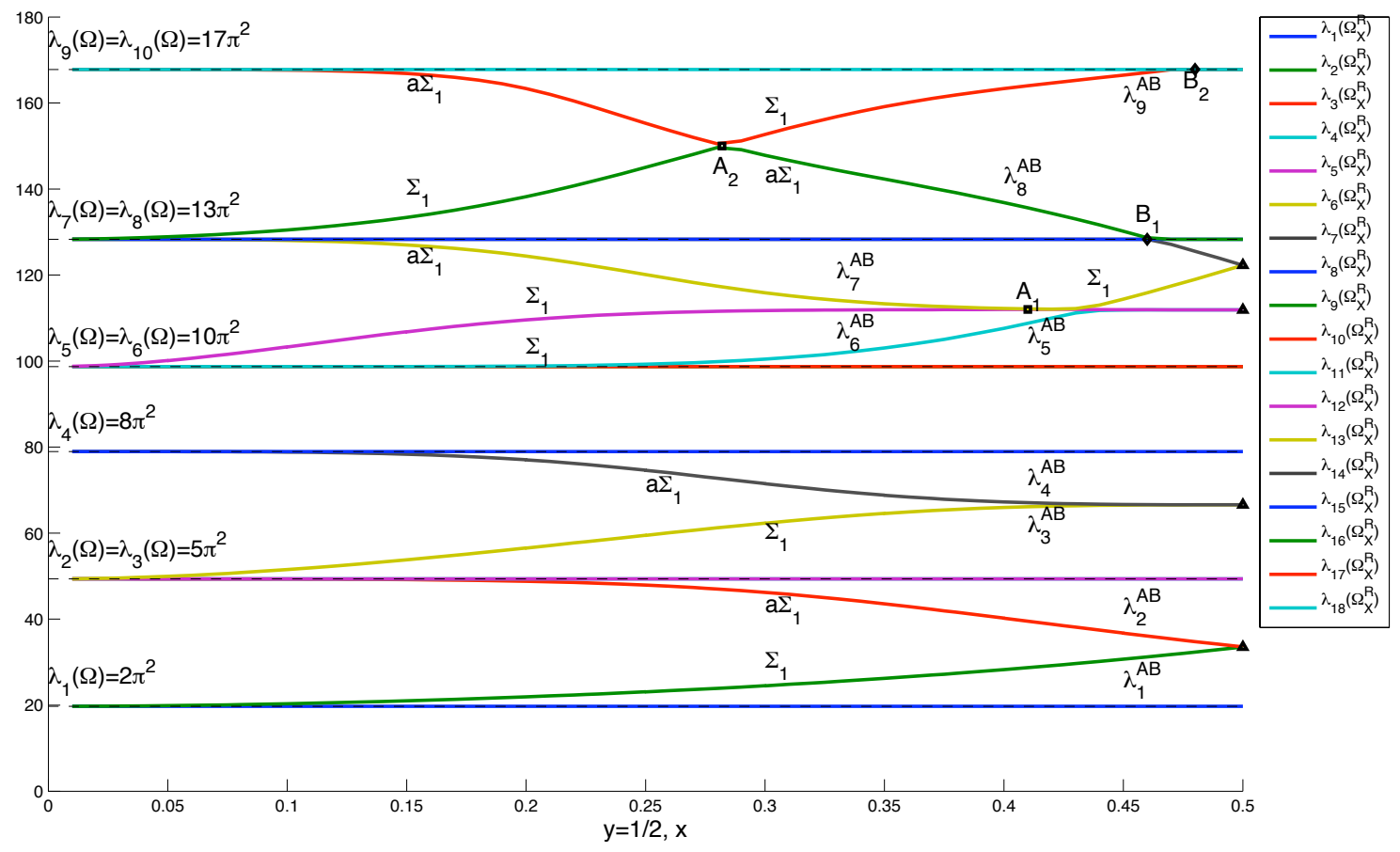

Figure 18: Moving the pole along the axis $y=1 / 2$.

(b) $x \mapsto \lambda_{2}\left(\dot{\Omega}_{X(x)}^{\mathcal{R}}\right)=\lambda_{1}^{\mathbf{A B} X(x)}$ is strictly increasing from $\left[0, \frac{1}{2}\right]$ onto $\left[\lambda_{1}(\Omega), \lambda_{1}^{\mathbf{A B C}}\right]$ and the eigenfunctions are symmetric. The equality between $\lambda_{2}\left(\dot{\Omega}_{X(x)}^{\mathcal{R}}\right)$ and $\lambda_{1}^{\mathbf{A B} X(x)}$ was proved in (3.1).

(c) $x \mapsto \lambda_{3}\left(\dot{\Omega}_{X(x)}^{\mathcal{R}}\right)=\lambda_{2}^{\mathbf{A B} X(x)}$ is strictly decreasing from $\left[0, \frac{1}{2}\right]$ onto $\left[\lambda_{2}^{\mathbf{A B} C}, \lambda_{2}(\Omega)\right]$ and the eigenfunctions are antisymmetric. The equality between $\lambda_{3}\left(\dot{\Omega}_{X(x)}^{\mathcal{R}}\right)$ and $\lambda_{2}^{\mathbf{A B} X(x)}$ was proved in (3.1).

(d) $\lambda_{4}\left(\dot{\Omega}_{X(x)}^{\mathcal{R}}\right)=\lambda_{5}\left(\dot{\Omega}_{X(x)}^{\mathcal{R}}\right)=\lambda_{2}(\Omega)$. This numerical observation is more accurate than the theoretical result deduced from $(3.2): \lambda_{4}\left(\dot{\Omega}_{X}^{\mathcal{R}}\right) \leq \lambda_{2}(\Omega)$.

(e) $x \mapsto \lambda_{6}\left(\dot{\Omega}_{X(x)}^{\mathcal{R}}\right)=\lambda_{3}^{\mathbf{A B} X(x)}$ is strictly increasing from $\left[0, \frac{1}{2}\right]$ onto $\left[\lambda_{3}(\Omega), \lambda_{3}^{\mathbf{A B} C}\right]$ and the eigenfunctions are symmetric.

(f) $x \mapsto \lambda_{7}\left(\dot{\Omega}_{X(x)}^{\mathcal{R}}\right)=\lambda_{4}^{\mathbf{A B} X(x)}$ is strictly decreasing from $\left[0, \frac{1}{2}\right]$ onto $\left[\lambda_{4}^{\mathbf{A B} C}, \lambda_{4}(\Omega)\right]$ and the eigenfunctions are antisymmetric.

(g) $\lambda_{8}\left(\dot{\Omega}_{X(x)}^{\mathcal{R}}\right)=\lambda_{4}(\Omega)$. We have proved in (3.8) that $\lambda_{8}\left(\dot{\Omega}_{X(x)}^{\mathcal{R}}\right) \leq \lambda_{4}(\Omega)$ and we observe that this upper-bound is actually an equality. We notice that there is a gap between $\lambda_{4}(\Omega)$ and $\lambda_{5}(\Omega)$ where there is no eigenvalue $\lambda_{k}\left(\dot{\Omega}_{X}^{\mathcal{R}}\right)$ for $X$ on the perpendicular bisector. This observation is no more true for poles on the diagonal (see Figure 22).

(h) $\lambda_{9}\left(\dot{\Omega}_{X(x)}^{\mathcal{R}}\right)=\lambda_{10}\left(\dot{\Omega}_{X(x)}^{\mathcal{R}}\right)=\lambda_{5}(\Omega)$.

(i) $x \mapsto \lambda_{11}\left(\dot{\Omega}_{X(x)}^{\mathcal{R}}\right)=\lambda_{5}^{\mathbf{A B} X(x)}$ is strictly increasing from $\left[0, \frac{1}{2}\right]$ onto $\left[\lambda_{5}(\Omega), \lambda_{5}^{\mathbf{A B} C}\right]$ and the eigenfunctions are symmetric. This observation shows that the theoretical upper-bound $\lambda_{11}\left(\dot{\Omega}_{X}^{\mathcal{R}}\right) \leq \lambda_{7}(\Omega)$ deduced from (3.5) can not be improved. 
(j) $x \mapsto \lambda_{12}\left(\dot{\Omega}_{X(x)}^{\mathcal{R}}\right)=\lambda_{6}^{\mathbf{A B} X(x)}$ is strictly increasing from $\left[0, a_{1}\right]$ onto $\left[\lambda_{6}(\Omega), \lambda_{6}^{\mathbf{A B} A_{1}}\right]$ and the eigenfunctions are symmetric. It is strictly decreasing from $\left[a_{1}, \frac{1}{2}\right]$ onto $\left[\lambda_{6}^{\mathbf{A B} C}, \lambda_{6}^{\mathbf{A B} A_{1}}\right]$ and the eigenfunctions are antisymmetric. This illustrates theoretical result deduced from (3.9): $\lambda_{12}\left(\dot{\Omega}_{X(x)}^{\mathcal{R}}\right) \leq \lambda_{7}(\Omega)$ and shows that this result is optimal.

(k) $x \mapsto \lambda_{13}\left(\dot{\Omega}_{X(x)}^{\mathcal{R}}\right)=\lambda_{7}^{\mathbf{A B} X(x)}$ is strictly decreasing from $\left[0, a_{1}\right]$ onto $\left[\lambda_{7}(\Omega), \lambda_{7}^{\mathbf{A B} A_{1}}\right]$ and the eigenfunctions are antisymmetric. It is strictly increasing from $\left[a_{1}, \frac{1}{2}\right]$ onto $\left[\lambda_{7}^{\mathbf{A B} C}, \lambda_{7}^{\mathbf{A B} A_{1}}\right]$ and the eigenfunctions are symmetric. We observe then that $\lambda_{13}\left(\dot{\Omega}_{X}^{\mathcal{R}}\right)$ can be bounded from above by $\lambda_{7}(\Omega)$ whereas we have proved in (3.6) the upper-bound by $\lambda_{9}(\Omega)$.

(l) $\lambda_{14}\left(\dot{\Omega}_{X(x)}^{\mathcal{R}}\right)$ equals $\lambda_{8}(\Omega)$ on $\left[0, b_{1}\right]$. It equals $\lambda_{8}^{\mathbf{A B} X(x)}$ on $\left[b_{1}, \frac{1}{2}\right]$ and is strictly decreasing from $\left[b_{1}, \frac{1}{2}\right]$ onto $\left[\lambda_{8}^{\mathbf{A B} C}, \lambda_{8}(\Omega)\right]$ with antisymmetric eigenfunctions.

(m) $\lambda_{15}\left(\dot{\Omega}_{X(x)}^{\mathcal{R}}\right)=\lambda_{8}(\Omega)$.

(n) $\lambda_{16}\left(\dot{\Omega}_{X(x)}^{\mathcal{R}}\right)$ equals $\lambda_{8}^{\mathbf{A B} X(x)}$ on $\left[0, b_{1}\right]$ and $\lambda_{8}(\Omega)$ on $\left[b_{1}, \frac{1}{2}\right]$. It is strictly increasing from $\left[0, a_{2}\right]$ onto $\left[\lambda_{8}(\Omega), \lambda_{8}^{\mathbf{A B} A_{2}}\right]$ with symmetric eigenfunctions and strictly decreasing from $\left[a_{2}, b_{1}\right]$ onto $\left[\lambda_{8}(\Omega), \lambda_{8}^{\mathbf{A B} A_{2}}\right]$ with antisymmetric eigenfunctions.

(o) $\lambda_{17}\left(\dot{\Omega}_{X(x)}^{\mathcal{R}}\right)$ equals $\lambda_{9}^{\mathbf{A B} X(x)}$ on $\left[0, b_{2}\right]$ and $\lambda_{9}(\Omega)$ on $\left[b_{2}, \frac{1}{2}\right]$. It is strictly decreasing from $\left[0, a_{2}\right]$ onto $\left[\lambda_{9}^{\mathbf{A B} A_{2}}, \lambda_{9}(\Omega)\right]$ with antisymmetric eigenfunctions and strictly increasing from $\left[a_{2}, b_{2}\right]$ onto $\left[\lambda_{9}^{\mathbf{A B} A_{2}}, \lambda_{9}(\Omega)\right]$ with symmetric eigenfunctions.

\subsection{Exchange of symmetry and crossing points}

When moving the pole on one bisector, and for each eigenvalue of multiplicity 1 , the corresponding $K_{X}$-real eigenfunction should be either symmetric or antisymmetric with respect to $\Sigma_{1}^{c}$. Figure 18 suggests that there exists two poles $A_{1}=\left(a_{1}, \frac{1}{2}\right)$ and $A_{2}=\left(a_{2}, \frac{1}{2}\right)$ on the perpendicular bisector such that $\lambda_{12}\left(\dot{\Omega}_{A_{1}}^{\mathcal{R}}\right)$ and $\lambda_{16}\left(\dot{\Omega}_{A_{2}}^{\mathcal{R}}\right)$ are eigenvalues of multiplicity 2. Taking the Aharonov-Bohm point of view, this corresponds to a crossing between $\lambda_{6}^{\mathbf{A B} X(x)}$ and $\lambda_{7}^{\mathbf{A B} X(x)}$ for $x=a_{1}$, with $\left.a_{1} \in\right] \frac{42}{100}, \frac{43}{100}\left[\right.$ and to a crossing between $\lambda_{8}^{\mathbf{A B} X(x)}$ and $\lambda_{9}^{\mathrm{AB} X(x)}$ at $x=a_{2}$, with $\left.a_{2} \in\right] \frac{28}{100}, \frac{29}{100}[$. The nodal sets of the corresponding eigenfunctions are given in Figures 19 and 20. The first line of the figure gives the eigenvalues $\lambda_{6}^{\mathbf{A B X}}$ (resp. $\lambda_{8}^{\mathbf{A B} X}$ ) and the nodal set of the associated eigenfunction and the second line $\lambda_{7}^{\mathrm{AB} X}$ (resp. $\lambda_{9}^{\mathbf{A B} X}$ ) and the corresponding nodal set for $X$ along the perpendicular bisector and close to $A_{1}$ (resp. $A_{2}$ ).

We can verify on each line of the two figures, that there exists an exchange of symmetry ${ }^{3}$ between the third column and the fourth column predicting the existence of the points $A_{1}$ and $A_{2}$.

We observe in the second line and fifth column of Figure 19 one nodal line with no selfcrossing. This is an artefact produced by our algorithm to detect the nodal lines and the accuracy of our computation. There is actually a crossing between a closed line and the horizontal segment joining the pole to the boundary of the square.

${ }^{3}$ Look at the horizontal nodal line joining the pole to the boundary! 


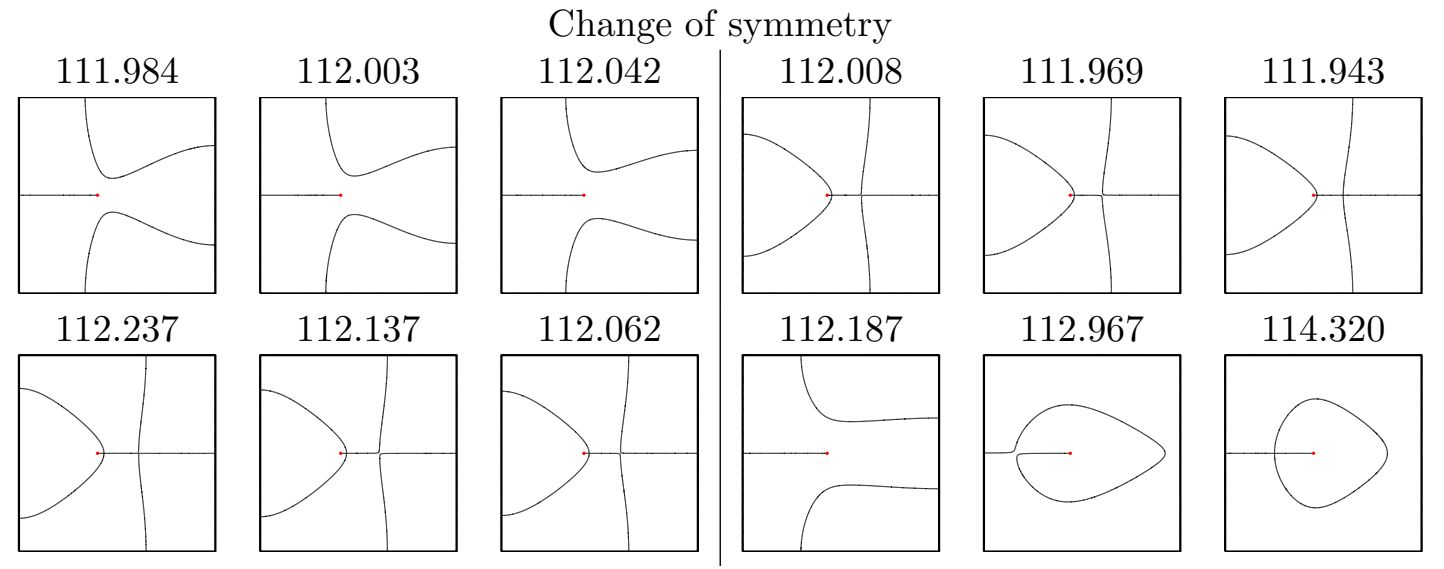

Figure 19: Nodal set for the eigenfunctions associated with $\lambda_{k}^{\mathbf{A B} X(x)}, k=6,7$ for poles $X=\left(\frac{i}{100}, \frac{1}{2}\right), 40 \leq i \leq 45$.

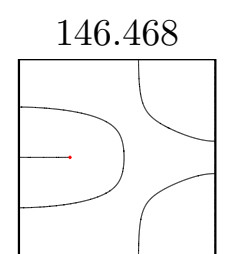

153.514

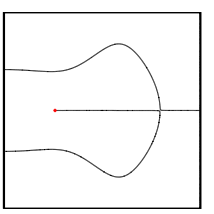

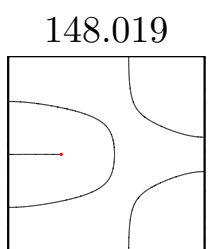

151.934

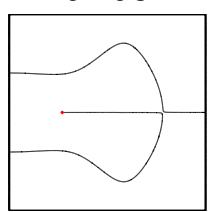

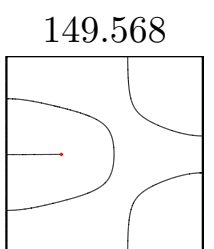

150.454

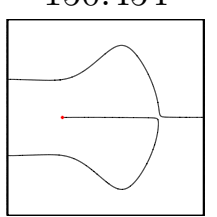

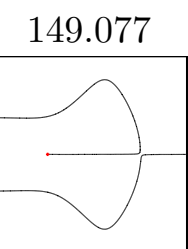

151.106

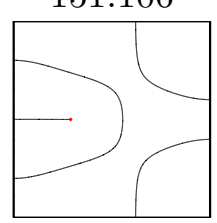

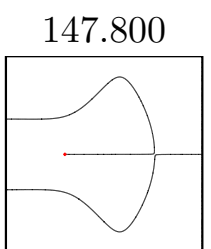

152.615

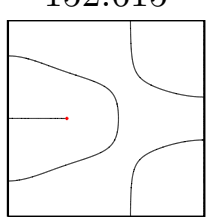

146.591

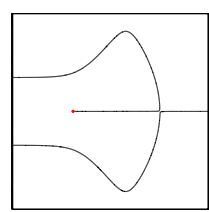

154.077

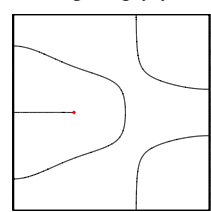

Figure 20: Nodal set for the eigenfunctions associated with $\lambda_{k}^{A B X(x)}, k=8,9$ for poles $X=\left(\frac{i}{100}, \frac{1}{2}\right), 26 \leq i \leq 31$.

\subsection{Nodal deformation : an example}

The last column of Figure A3 gives the nodal set for the eigenfunction associated with the fifth eigenvalue of the $\mathbf{A B} X$-Hamiltonian for poles $X=\left(\frac{i}{20}, \frac{1}{2}\right), i=1, \ldots, 10$, on the perpendicular bisector of one side of the square. The deformation mechanism for the nodal set is represented in Figure 21. Between the fourth and fifth figures, we have a nodal structure where there are two double points at the boundary.

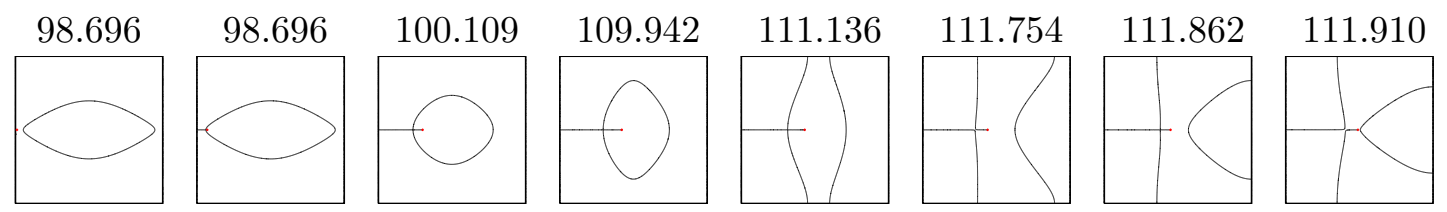

Figure 21: Nodal set for the 5-th eigenfunction of the AB-Hamiltonian with poles $X=$ $\left(\frac{i}{100}, \frac{1}{2}\right), i=1,7,30,42,43,44,45,49$. 


\section{Moving the pole on the diagonal}

\subsection{Analysis of the symmetries}

As for the case of poles along the perpendicular bisector, we can use the symmetry along the diagonal to have additional information about the spectrum.

The square is invariant under the symmetry

$$
\sigma_{2}(x, y)=(y, x) .
$$

We consider the antilinear operator $\Sigma_{2}^{c}=\Gamma \Sigma_{2}$ where $\Sigma_{2}$ is associated with $\sigma_{2}$ by the relation $\Sigma_{2} u(x, y)=u(y, x)$.

We use this symmetry to give an orthogonal decomposition of $L_{K}^{2}=L_{K_{X}}^{2}$ :

$$
L_{K}^{2}=L_{K, \Sigma_{2}}^{2} \oplus L_{K, a \Sigma_{2}}^{2}
$$

where $L_{K, \Sigma_{2}}^{2}$ and $L_{K, a \Sigma_{2}}^{2}$ are respectively symmetric and antisymmetric with respect to $\Sigma_{2}^{c}$. As in [3, Lemma 5.6], we can prove that, with $X=\left(x_{0}, x_{0}\right)$,

- if $u \in C^{\infty}\left(\dot{\Omega}_{X}\right) \cap L_{K, \Sigma_{2}}^{2}$, then the nodal set of $u$ contains $\left\{(x, x), 0<x<x_{0}\right\}$;

- if $u \in C^{\infty}\left(\dot{\Omega}_{X}\right) \cap L_{K, a \Sigma_{2}}^{2}$, then the nodal set of $u$ contains $\left\{(x, x), x_{0}<x<1\right\}$.

Dealing with a mixed Dirichlet-Neumann condition on the half-domain, we deduce that the eigenvalues for which the eigenfunctions are symmetric are increasing with respect to $x_{0}$ whereas the eigenvalues for which the eigenfunctions are antisymmetric are decreasing with respect to $x_{0}$.

\subsection{Spectral variation}

Figure 22 gives the eigenvalues for poles along the diagonal line of the square $x=y$ with $0 \leq x \leq 1 / 2$. We observe that the eigenvalues $\lambda_{k}^{\mathbf{A B} \check{X}(x)}$ with $\check{X}(x)=(x, x)$ are increasing with $x \in\left[0, \frac{1}{2}\right]$ for $k=1,3$ and decreasing for $k=2$.

We introduce $C_{j}=\check{X}\left(c_{j}\right), D_{j}=\check{X}\left(d_{j}\right)$ specific crossing points appearing on the figure. The below mentioned symmetry (resp. antisymmetry) is in this section with respect to $\Sigma_{2}^{c}$ and denoted by $\Sigma_{2}$ (resp. $a \Sigma_{2}$ ) on the Figure 22. Then, we observe:

(a) $x \mapsto \lambda_{1}\left(\dot{\Omega}_{\tilde{X}(x)}^{\mathcal{R}}\right)$ equals $\lambda_{1}(\Omega)$, in adequation with (3.1).

(b) $x \mapsto \lambda_{2}\left(\dot{\Omega}_{\tilde{X}(x)}^{\mathcal{R}}\right)=\lambda_{1}^{\mathbf{A B} \check{X}(x)}$ is strictly increasing from $\left[0, \frac{1}{2}\right]$ onto $\left[\lambda_{1}(\Omega), \lambda_{1}^{\mathbf{A B} C}\right]$ and the eigenfunctions are symmetric. The equality between $\lambda_{2}\left(\dot{\Omega}_{\check{X}(x)}^{\mathcal{R}}\right)$ and $\lambda_{1}^{\mathbf{A B} \check{X}(x)}$ observed numerically was rigourously proved in (3.1).

(c) $x \mapsto \lambda_{3}\left(\dot{\Omega}_{\check{X}(x)}^{\mathcal{R}}\right)=\lambda_{2}^{\mathbf{A B} \check{X}(x)}$ is strictly decreasing from $\left[0, \frac{1}{2}\right]$ onto $\left[\lambda_{2}^{\mathbf{A B} C}, \lambda_{2}(\Omega)\right]$ and the eigenfunctions are antisymmetric. The equality between $\lambda_{3}\left(\dot{\Omega}_{\tilde{X}(x)}^{\mathcal{R}}\right)$ and $\lambda_{2}^{\mathbf{A B} \check{X}(x)}$ observed numerically was also rigourously proved in (3.1). 


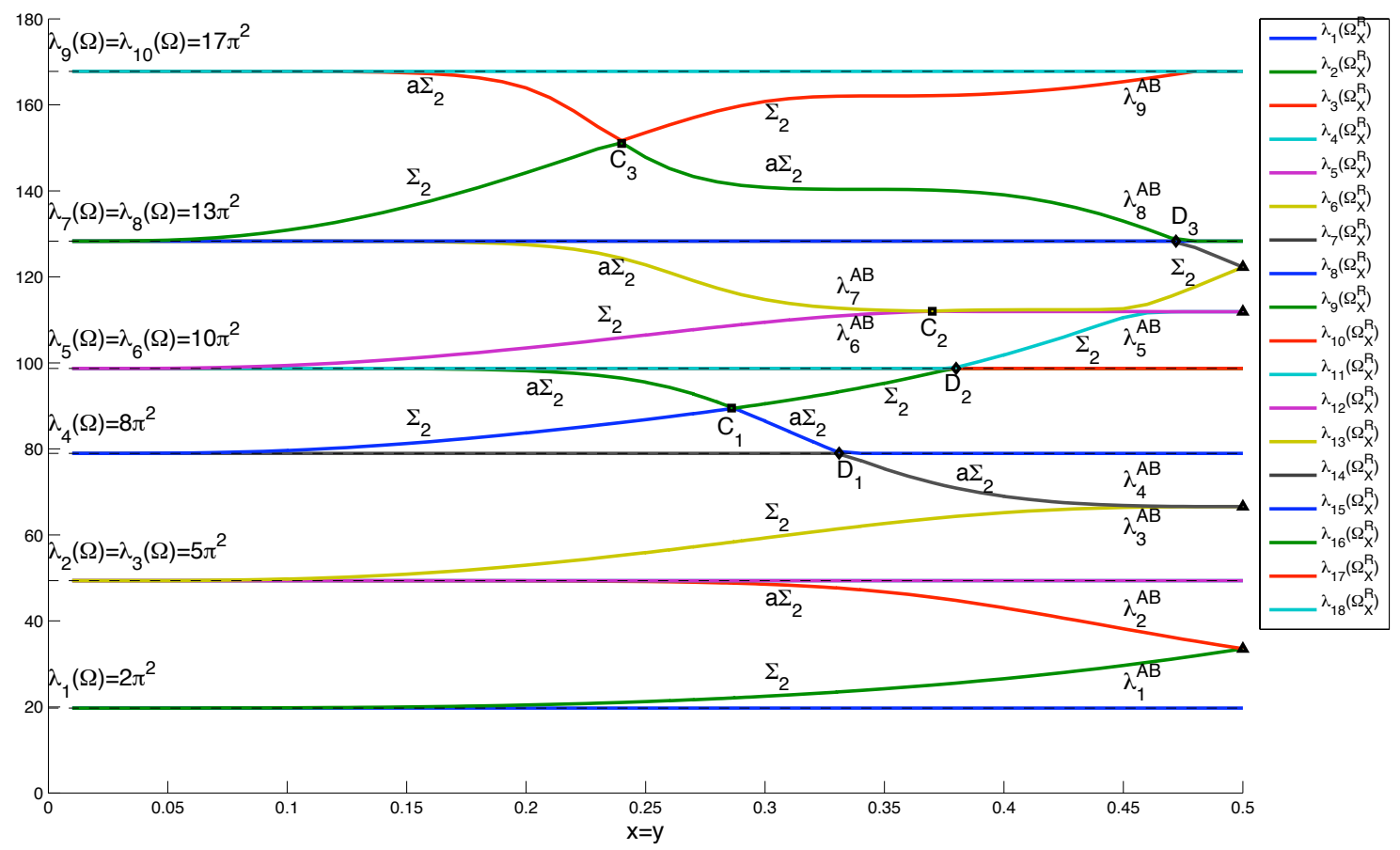

Figure 22: Moving the poles on the diagonal.

(d) $\lambda_{4}\left(\dot{\Omega}_{\tilde{X}(x)}^{\mathcal{R}}\right)=\lambda_{5}\left(\dot{\Omega}_{\tilde{X}(x)}^{\mathcal{R}}\right)=\lambda_{2}(\Omega)$. Theoretically, we have proved, see (3.2), that $\lambda_{4}\left(\dot{\Omega}_{\tilde{X}(x)}^{\mathcal{R}}\right) \leq \lambda_{2}(\Omega)$. This relation seems to be an equality, see Subsection 4.3.

(e) $x \mapsto \lambda_{6}\left(\dot{\Omega}_{\tilde{X}(x)}^{\mathcal{R}}\right)=\lambda_{3}^{\mathbf{A B} \check{X}(x)}$ is strictly increasing from $\left[0, \frac{1}{2}\right]$ onto $\left[\lambda_{3}(\Omega), \lambda_{3}^{\mathbf{A B} C}\right]$ and the eigenfunctions are symmetric.

(f) $x \mapsto \lambda_{7}\left(\dot{\Omega}_{\tilde{X}(x)}^{\mathcal{R}}\right)$ equals $\lambda_{4}(\Omega)$ on $\left[0, d_{1}\right]$ and $\lambda_{4}^{\mathbf{A B} \check{X}(x)}$ on $\left[d_{1}, \frac{1}{2}\right]$ where it is strictly decreasing onto $\left[\lambda_{4}^{\mathbf{A B C}}, \lambda_{4}(\Omega)\right]$ and the eigenfunctions are antisymmetric. This numerical computations show that the theoretical estimate $\lambda_{7}\left(\dot{\Omega}_{X}^{\mathcal{R}}\right) \leq \lambda_{4}(\Omega)$, deduced from (3.3) is optimal.

(g) $\lambda_{8}\left(\dot{\Omega}_{\tilde{X}(x)}^{\mathcal{R}}\right)$ equals $\lambda_{4}^{\mathrm{AB} \check{X}(x)}$ on $\left[0, d_{1}\right]$ and $\lambda_{4}(\Omega)$ on $\left[d_{1}, \frac{1}{2}\right]$. It is strictly increasing from $\left[0, c_{1}\right]$ onto $\left[\lambda_{4}(\Omega), \lambda_{4}\left(\dot{\Omega}_{C_{1}}^{\mathcal{R}}\right)\right]$ with symmetric eigenfunctions and strictly decreasing from $\left[c_{1}, d_{1}\right]$ onto $\left[\lambda_{4}(\Omega), \lambda_{4}\left(\dot{\Omega}_{C_{1}}^{\mathcal{R}}\right)\right]$ with antisymmetric eigenfunctions. This illustrates that (3.4) is optimal.

(h) $\lambda_{9}\left(\dot{\Omega}_{\tilde{X}(x)}^{\mathcal{R}}\right)$ equals $\lambda_{5}^{\mathbf{A B} \check{X}(x)}$ on $\left[0, d_{2}\right]$ and $\lambda_{5}(\Omega)$ on $\left[d_{2}, \frac{1}{2}\right]$. It is strictly decreasing from $\left[0, c_{1}\right]$ onto $\left[\lambda_{4}\left(\dot{\Omega}_{C_{1}}^{\mathcal{R}}\right), \lambda_{5}(\Omega)\right]$ with antisymmetric eigenfunctions and strictly increasing from $\left[c_{1}, d_{1}\right]$ onto $\left[\lambda_{4}\left(\dot{\Omega}_{C_{1}}^{\mathcal{R}}\right), \lambda_{5}(\Omega)\right]$ with symmetric eigenfunctions.

(i) $\lambda_{10}\left(\dot{\Omega}_{\tilde{X}(x)}^{\mathcal{R}}\right)=\lambda_{5}(\Omega)$.

(j) $x \mapsto \lambda_{11}\left(\dot{\Omega}_{\check{X}(x)}^{\mathcal{R}}\right)$ equals $\lambda_{5}(\Omega)$ on $\left[0, d_{2}\right]$ and $\lambda_{5}^{\mathbf{A B} \check{X}(x)}$ on $\left[d_{2}, \frac{1}{2}\right]$ where it is strictly increasing onto $\left[\lambda_{5}(\Omega), \lambda_{5}^{\mathrm{AB} C}\right]$ and the eigenfunctions are symmetric. This illustrates the fact that relation (3.5) is optimal. 
(k) $x \mapsto \lambda_{12}\left(\dot{\Omega}_{\check{X}(x)}^{\mathcal{R}}\right)=\lambda_{6}^{\mathbf{A B} \check{X}(x)}$ is strictly increasing from $\left[0, c_{2}\right]$ onto $\left[\lambda_{6}(\Omega), \lambda_{6}^{\mathbf{A B} C_{2}}\right]$ and the eigenfunctions are symmetric. It is strictly decreasing from $\left[c_{2}, \frac{1}{2}\right]$ onto $\left[\lambda_{6}^{\mathbf{A B} C}, \lambda_{6}^{\mathbf{A B} C_{2}}\right]$ and the eigenfunctions are antisymmetric.

(l) $x \mapsto \lambda_{13}\left(\dot{\Omega}_{\tilde{X}(x)}^{\mathcal{R}}\right)=\lambda_{7}^{\mathbf{A B} \check{X}(x)}$ is strictly decreasing from $\left[0, c_{2}\right]$ onto $\left[\lambda_{7}(\Omega), \lambda_{7}^{\mathbf{A B} C_{2}}\right]$ and the eigenfunctions are antisymmetric. It is strictly increasing from $\left[c_{2}, \frac{1}{2}\right]$ onto $\left[\lambda_{7}^{\mathbf{A B} C}, \lambda_{7}^{\mathbf{A B} C_{2}}\right]$ and the eigenfunctions are symmetric. We then observe $\lambda_{13}\left(\dot{\Omega}_{\tilde{X}(x)}^{\mathcal{R}}\right) \leq$ $\lambda_{7}(\Omega)$ whereas we have proved the weaker upper-bound by $\lambda_{9}(\Omega)$ in $(3.6)$.

(m) $\lambda_{14}\left(\dot{\Omega}_{\tilde{X}(x)}^{\mathcal{R}}\right)$ equals $\lambda_{7}(\Omega)$ on $\left[0, d_{3}\right]$. It equals $\lambda_{8}^{\mathbf{A B} \check{X}(x)}$ on $\left[d_{3}, \frac{1}{2}\right]$ and is strictly decreasing from $\left[d_{3}, \frac{1}{2}\right]$ onto $\left[\lambda_{8}^{\mathbf{A B} C}, \lambda_{8}(\Omega)\right]$ with antisymmetric eigenfunctions.

(n) $\lambda_{15}\left(\dot{\Omega}_{\tilde{X}(x)}^{\mathcal{R}}\right)=\lambda_{8}(\Omega)$.

(o) $\lambda_{16}\left(\dot{\Omega}_{\tilde{X}(x)}^{\mathcal{R}}\right)$ equals $\lambda_{8}^{\mathbf{A B} \check{X}(x)}$ on $\left[0, d_{3}\right]$ and $\lambda_{8}(\Omega)$ on $\left[d_{3}, \frac{1}{2}\right]$. It is strictly increasing from $\left[0, c_{3}\right]$ onto $\left[\lambda_{8}(\Omega), \lambda_{8}^{\mathbf{A B} C_{3}}\right]$ with symmetric eigenfunctions and strictly decreasing from $\left[c_{3}, d_{3}\right]$ onto $\left[\lambda_{8}(\Omega), \lambda_{8}^{\mathbf{A B} C_{3}}\right]$ with antisymmetric eigenfunctions.

(p) $\lambda_{17}\left(\dot{\Omega}_{\check{X}(x)}^{\mathcal{R}}\right)$ equals $\lambda_{9}^{\mathbf{A B} \check{X}(x)}$ on $\left[0, \frac{1}{2}\right]$. It is strictly decreasing from $\left[0, c_{3}\right]$ onto $\left[\lambda_{9}^{\mathbf{A B} C_{3}}, \lambda_{9}(\Omega)\right]$ with antisymmetric eigenfunctions and strictly increasing from $\left[c_{3}, \frac{1}{2}\right]$ onto $\left[\lambda_{9}^{\mathbf{A B} C_{3}}, \lambda_{9}(\Omega)\right]$ with symmetric eigenfunctions. We observe that $\lambda_{17}\left(\dot{\Omega}_{\tilde{X}(x)}^{\mathcal{R}}\right) \leq$ $\lambda_{9}(\Omega)$ whereas we have proved in (3.7) the upper-bound by $\lambda_{11}(\Omega)$.

\subsection{Exchange of symmetry}

As in the case of the symmetry with respect to the bisector, when moving the pole on one diagonal, and for each eigenvalue of multiplicity 1 , the corresponding $K_{X}$-real eigenfunction should be either symmetric or antisymmetric with respect to the quantized symmetry with respect to the diagonal (see [3]). Figure 22 suggests that there are 3 points, $C_{1}, C_{2}$ and $C_{3}$ on the diagonal such that $\lambda_{8}\left(\dot{\Omega}_{C_{1}}^{\mathcal{R}}\right), \lambda_{12}\left(\dot{\Omega}_{C_{2}}^{\mathcal{R}}\right)$ and $\lambda_{16}\left(\dot{\Omega}_{C_{3}}^{\mathcal{R}}\right)$ are eigenvalues of multiplicity 2. This corresponds to a crossing between $\lambda_{4}^{\mathbf{A B} \check{X}(x)}$ and $\lambda_{5}^{\mathbf{A B} \check{X}(x)}$ at $x=c_{1}$, with $\left.c_{1} \in\right] \frac{28}{100}, \frac{29}{100}$ [. Similarly, there is a crossing between $\lambda_{6}^{\mathbf{A B} \check{X}(x)}$ and $\lambda_{7}^{\mathbf{A B} \check{X}(x)}$ at $x=c_{2}$, with $\left.c_{2} \in\right] \frac{36}{100}, \frac{37}{100}\left[\right.$, and also between $\lambda_{8}^{\mathbf{A B} \check{X}(x)}$ and $\lambda_{9}^{\mathbf{A B} \check{X}(x)}$ at $x=c_{3}$, with $\left.c_{3} \in\right] \frac{23}{100}, \frac{24}{100}[$. The nodal set of the corresponding eigenfunctions are given in Figures 23, 24 and 25. We can verify that there exists an exchange of symmetry permitting to predict the existence of the points $C_{1}, C_{2}$ and $C_{3}$.

The nodal lines have to respect the symmetry according to the diagonal line. Consequently, in each case, there exists a nodal line touching the point $(0,0)$ or $(1,1)$. In some picture, the touching point is not exactly one of these points but it is quite difficult to detect accurately these lines near the boundary because the eigenfunction vanishes at the boundary. 


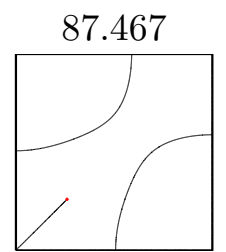

94.276

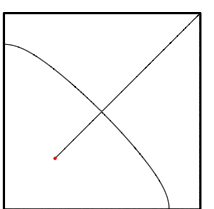

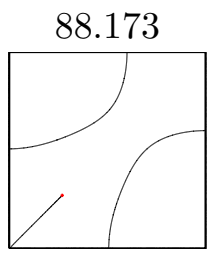

92.744

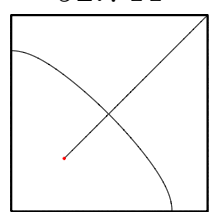

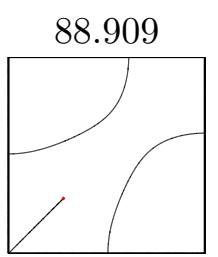

90.880

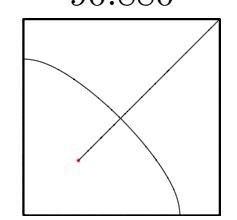

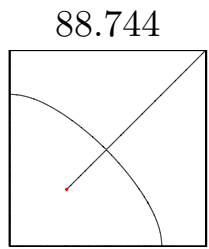

89.677

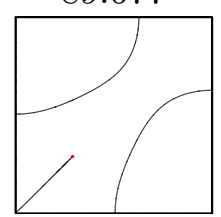

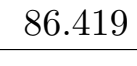

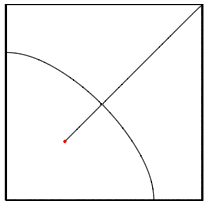

90.481

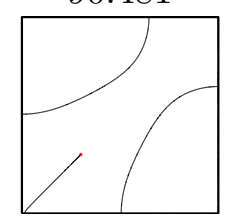

84.023

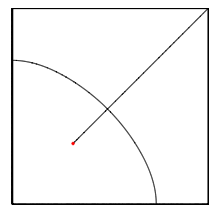

91.325

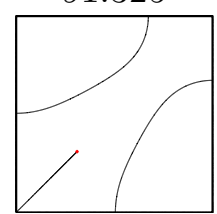

Figure 23: Nodal set for the eigenfunctions associated with $\lambda_{k}^{\mathrm{AB} X}, k=4,5$ for poles $X=(i, i) / 100,26 \leq i \leq 31$.

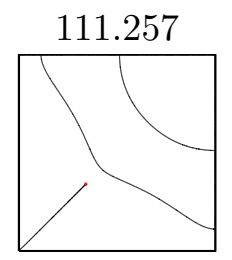

112.426

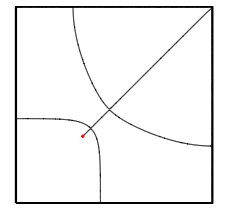

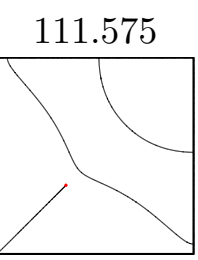

112.223

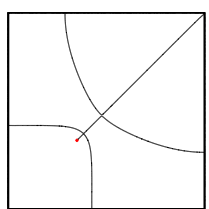

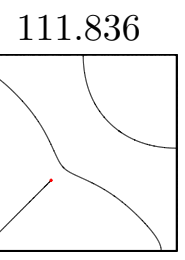

112.093

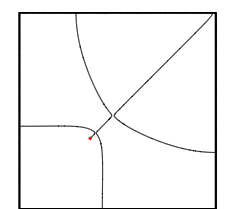

112.013

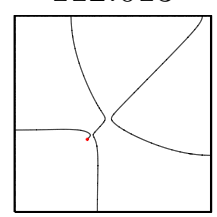

112.037

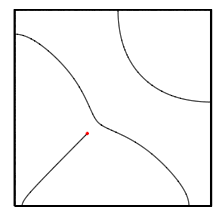

111.968

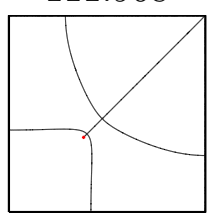

112.185

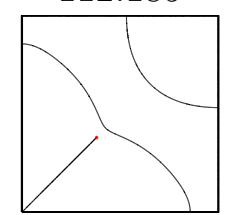

111.945

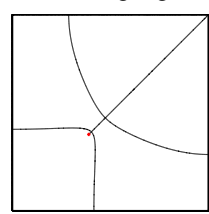

112.284

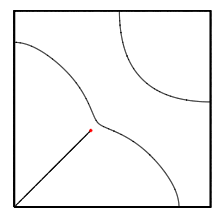

Figure 24: Nodal set for the eigenfunctions associated with $\lambda_{k}^{\mathbf{A B} X}, k=6,7$ for poles $X=(i, i) / 100,34 \leq i \leq 39$.

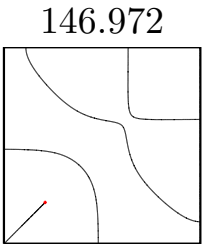

161.593

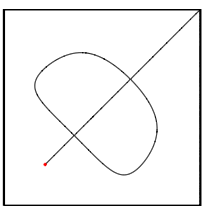

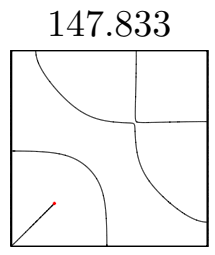

158.489

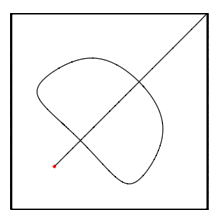

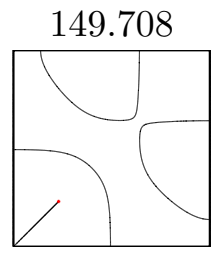

154.785

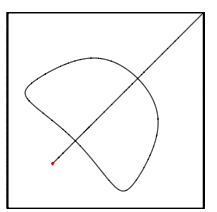

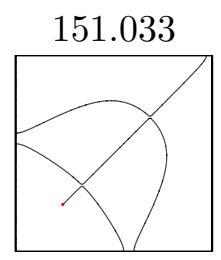

151.601

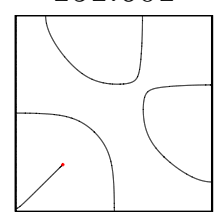

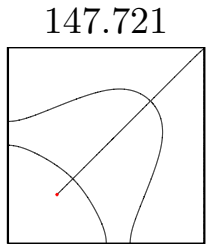

153.467

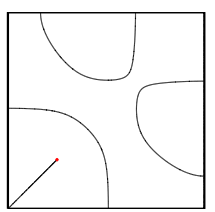

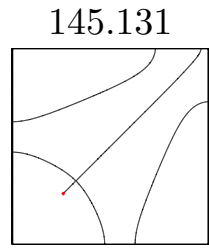

155.275

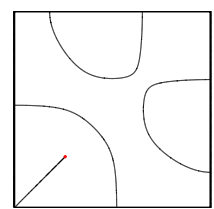

Figure 25: Nodal set for the eigenfunctions associated with $\lambda_{k}^{\mathbf{A B} X}, k=8,9$ for poles $X=(i, i) / 100,21 \leq i \leq 26$.

\subsection{Moving the pole without respecting the symmetries of the square}

Figure 26 gives the eigenvalues $\lambda_{k}\left(\dot{\Omega}_{X}^{\mathcal{R}}\right)$ for $1 \leq k \leq 12$ when the pole $X$ belongs to the line $y=\frac{1}{4}+\frac{x}{2}$. We choose this axis to exhibit a case without symmetry and we notice that the 
AB-eigenvalues $\lambda_{k}^{\mathbf{A B} X}$ are no longer monotone with respect to $x$ when $X=\left(x, \frac{1}{4}+\frac{x}{2}\right)$. The result should be the same for any arbitrary line (except the perpendicular bisector and the diagonal). We choose to present the simulations for this line because this line contains enough points in $\mathcal{P}$ to use the previous numerical computations.

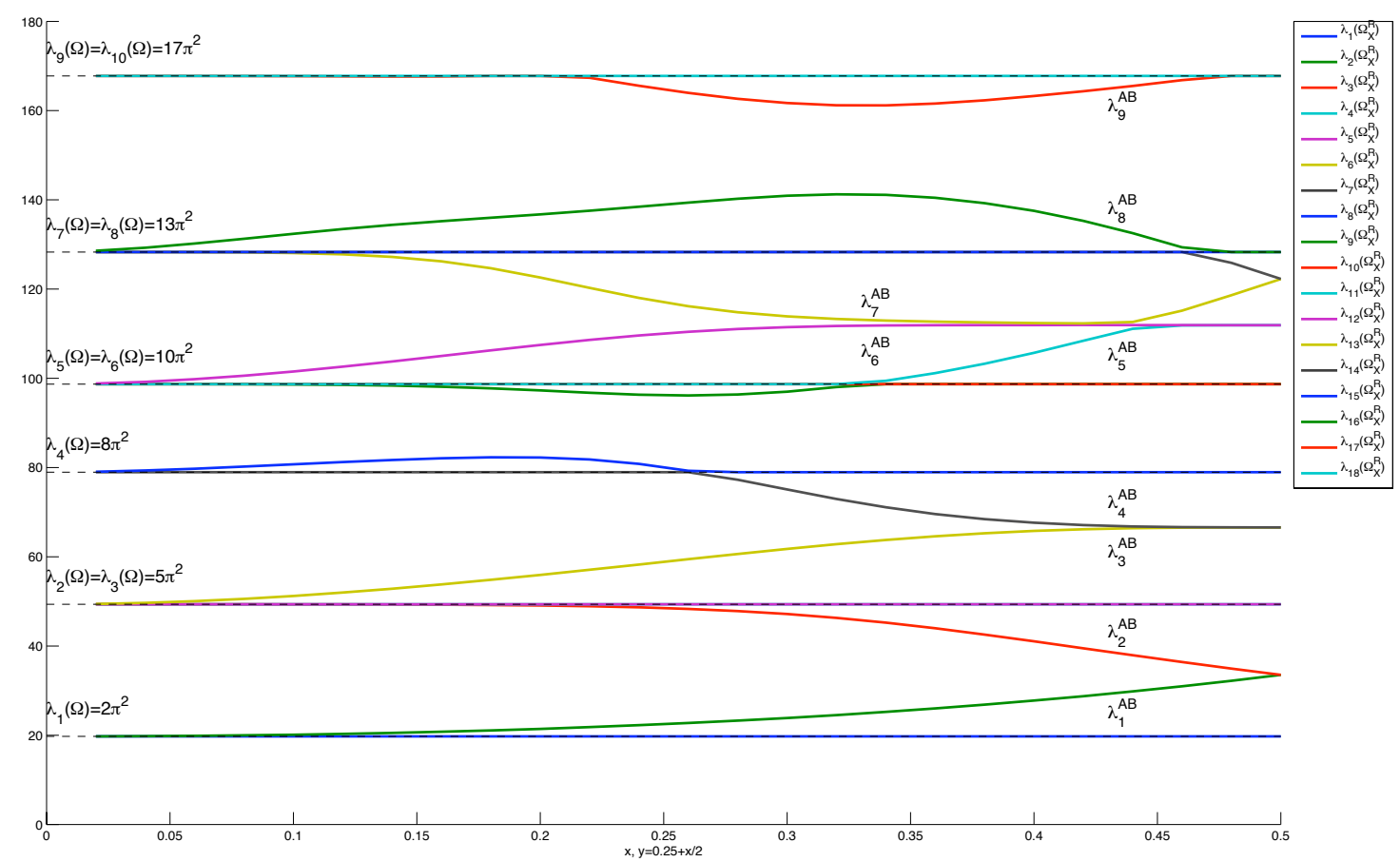

Figure 26: Moving the pole along the axis $y=\frac{1}{4}+\frac{x}{2}, 0 \leq x \leq \frac{1}{2}$.

It would be interesting to make computations for a finer grid of $X=\left(x, \frac{1}{4}+\frac{x}{2}\right)$ for $x$ around 0.44 to detect possible crossings between $\lambda_{5}^{\mathbf{A B}}, \lambda_{6}^{\mathbf{A B}}$ and $\lambda_{7}^{\mathrm{AB}}$.

\section{Nodal sets and minimal partitions}

The analysis of $\lambda_{5}^{\mathrm{AB} X}$ and $\lambda_{6}^{\mathrm{AB} X}$ leads us to guess numerically a double eigenvalue at the center. As for the pairs $\lambda_{3}^{\mathbf{A B X}}$ and $\lambda_{4}^{\mathbf{A B X}}$ which lead us to the family of candidates for the minimal 3-partitions of the square (see Subsection 4.4), we are led to produce a candidate for a minimal 5-partition for the square, with the property that it is minimal inside the class of 5-partitions which can be lifted to $\dot{\Omega}_{C}^{\mathcal{R}}$. Although $\lambda_{5}^{\mathrm{AB} C}=\lambda_{11}\left(\dot{\Omega}_{C}^{\mathcal{R}}\right)$, which is not Courant-sharp, we observe that it is Courant-sharp for the AB-Hamiltonian.

The eigenfunctions computed with the double covering approach do not produce a 5 -partition actually a small perturbation of it with only three nodal sets in $\dot{\Omega}_{C}$, but we can have a better insight by taking the Dirichlet-Neumann approach along the bisector orthogonal to one side: we compute the 5-th eigenfunction of the Dirichlet Laplacian on the square with Dirichlet condition on $\left\{\left(x, \frac{1}{2}\right), \frac{1}{2}<x<1\right\}$, see Figure 28 .

This time, neither numerics nor theory is giving the existence of a continuous family of 5-partitions. Actually, one knows from elementary results on the perturbation of harmonic 
polynomials of order 2 that the perpendicular crossing of two lines will generically disappear by perturbation.

In the 2D eigenspace of $\lambda_{11}\left(\dot{\Omega}_{C}^{\mathcal{R}}\right)$ only four eigenfunctions seem to lead to a configuration on one sheet with five nodal domains. The other eigenfunctions seem to have four symmetric (for the deck map) pairs of domains. These eigenfunctions are symmetric or antisymmetric with respect of one of the four bisectors of the square (see Figure 27). Looking at the linear combination $t u_{11}+(1-t) u_{12}$ of the eigenfunction associated with $\lambda_{11}\left(\dot{\Omega}_{C}^{\mathcal{R}}\right)$ and $\lambda_{12}\left(\dot{\Omega}_{C}^{\mathcal{R}}\right)$, we observe in Figure 27 that the triple point is very unstable and only appears for $t \simeq \frac{96}{200}$ and $t \simeq \frac{194}{200}$ when we consider $0 \leq t \leq 1$.

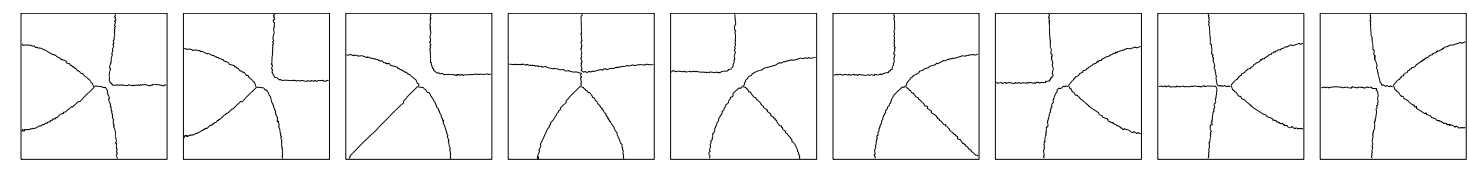

Figure 27: Nodal sets of the linear combination of $u_{11}$ and $u_{12}$ $t u_{11}+(1-t) u_{12}$ with $t=\frac{1}{200}(0,25,55,96,125,138,175,194,200)$.

Of course it is interesting to compare with what can be obtained by looking at other topological types for the 5-minimal partitions. We recall that these types can be classified by using Euler formula (see [11] for the case of 3-partitions). Inspired [9], we look for a partition which has the symmetries of the square and four critical points. We get two types of models that we can reduce to a Dirichlet-Neumann problem on a triangle corresponding to the eighth of the square. Moving the Neumann boundary on one side like in [4] leads to two candidates. Numerical computations demonstrate a lower energy in one case which coincides with one of the pictures in [9] (see Figure 28).
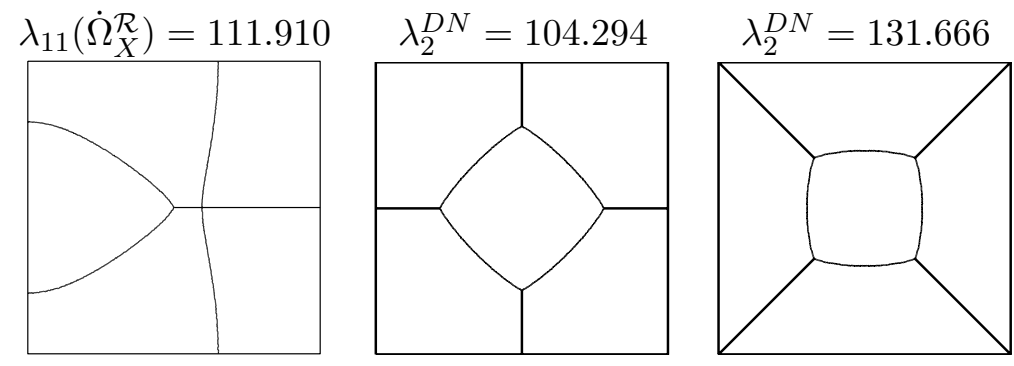

Figure 28: Three candidates for the 5-partition of the square.

\section{Remark 7.1}

Note that in the case of the disk a similar analysis leads to a different answer. The partition of the disk by five halfrays with equal angle has a lower energy than the minimal 5-partition with four singular points (see Figure 29). We note that, on the basis of standard computations (see for example (A1) and (A5) in [12], Appendix A) this energy corresponds to the eleventh eigenvalue of the Dirichlet problem on the double covering on the punctured disk (hence is not Courant sharp) but corresponds to the fifth of the Aharonov-Bohm spectrum of the disk. Hence it is Courant sharp in the sense developed in [13] (for the sphere) and shows the minimality of this 5-partition inside the minimal 5-partitions of the disk having a unique critical point at the center. 


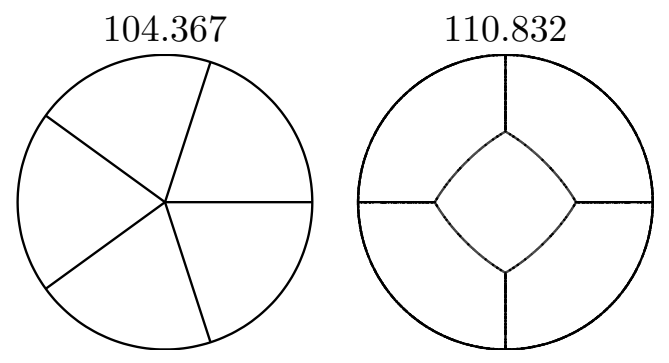

Figure 29: Two candidates for the 5-partition of the disk.

\section{Conclusion}

We have explored rather systematically how minimal partitions could be obtained by looking at nodal domains of a problem on the double covering of a punctured square. We have analyzed the behavior of the nodal set when moving the pole in the square. This has permitted to confirm the status of "main" candidate for some 3-partitions in the case of the square. This has also permitted to exhibit a natural candidate for a minimal 5-partition which finally appears to be less favorable than another partition with four critical points. This is a starting point for a program which can be developed in at least two directions :

- analyze other domains,

- do the same work by considering the double covering of a multi-punctured domain and moving the poles.

\section{References}

[1] B. Alziary, J. Fleckinger-Pellé, P. Takáč. Eigenfunctions and Hardy inequalities for a magnetic Schrödinger operator in $\mathbb{R}^{2}$. Math. Methods Appl. Sci. 26(13) (2003) 1093-1136.

[2] J. Berger, J. Rubinstein. On the zero set of the wave function in superconductivity. Comm. Math. Phys. 202(3) (1999) 621-628.

[3] V. Bonnaillie-Nö̈l, B. Helffer, T. Hoffmann-Ostenhof. Aharonov-bohm hamiltonians, isospectrality and minimal partitions. J. Phys. A: Math. Theor. 42(18) (2009) 185203.

[4] V. Bonnaillie-Nö̈l, B. Helffer, G. Vial. Numerical simulations for nodal domains and spectral minimal partitions. ESAIM Control Optim. Calc. Var. DOI: 10.1051/cocv:2008074 (2009).

[5] V. Bonnaillie-NoËL, G. Vial. Computations for nodal domains and spectral minimal partitions.

http://w3.bretagne.ens-cachan.fr/math/simulations/MinimalPartitions (2007).

[6] M. Conti, S. Terracini, G. Verzini. An optimal partition problem related to nonlinear eigenvalues. J. Funct. Anal. 198(1) (2003) 160-196. 
[7] M. Conti, S. Terracini, G. Verzini. On a class of optimal partition problems related to the Fučík spectrum and to the monotonicity formulae. Calc. Var. Partial Differential Equations 22(1) (2005) 45-72.

[8] M. Conti, S. Terracini, G. Verzini. A variational problem for the spatial segregation of reaction-diffusion systems. Indiana Univ. Math. J. 54(3) (2005) 779-815.

[9] O. Cybulski, V. Babin, R. HoŁyst. Minimization of the Renyi entropy production in the space-partitioning process. Phys. Rev. E (3) 71(4) (2005) 046130, 10.

[10] B. Helffer, M. Hoffmann-Ostenhof, T. Hoffmann-Ostenhof, M. P. Owen. Nodal sets for groundstates of Schrödinger operators with zero magnetic field in nonsimply connected domains. Comm. Math. Phys. 202(3) (1999) 629-649.

[11] B. Helffer, T. Hoffmann-Ostenhof. On spectral minimal partitions : new properties and applications to the disk. To appear in Proceedings of the conference in Montreal. CRM Lectures (2009).

[12] B. Helffer, T. Hoffmann-Ostenhof, S. Terracini. Nodal domains and spectral minimal partitions. Ann. Inst. H. Poincaré Anal. Non Linéaire 26 (2009) 101138.

[13] B. Helffer, T. Hoffmann-Ostenhof, S. Terracini. On spectral minimal partitions : the case of the sphere. Around the Research of Vladimir Maz'ya III, International Math. Series 13 (2010) 153-178.

[14] L. Hillairet, C. Judge. The eigenvalues of the Laplacian on domains with small slits. arXiv : 0802.259\%. To appear in Trans. AMS (2009).

[15] D. Jakobson, M. Levitin, N. Nadirashvili, I. Polterovich. Spectral problems with mixed Dirichlet-Neumann boundary conditions: isospectrality and beyond. $J$. Comput. Appl. Math. 194(1) (2006) 141-155.

[16] M. Levitin, L. Parnovski, I. Polterovich. Isospectral domains with mixed boundary conditions. J. Phys. A 39(9) (2006) 2073-2082.

[17] D. Martin. MÉLina, bibliothèque de calculs éléments finis. http://perso.univ-rennes1.fr/daniel.martin/melina (2007).

[18] B. Noris, S. Terracini. Nodal sets of magnetic Schrödinger operators of AharonovBohm type and energy minimizing partitions. Preprint (2009).

[19] B. Noris, S. Terracini. In preparation (2009).

[20] J. R. Shewchuk. Triangle: A two-dimensional quality mesh generator and Delaunay triangulator. http://www.cs.cmu.edu/ quake/triangle.html (2005). 


\section{Appendix. Additional numerics for the first Aharonov-Bohm eigenvalues}

Figures A1-A4 give the nodal set for the eigenfunctions associated with $\lambda_{k}^{\mathbf{A B}}\left(\dot{\Omega}_{X}\right)$ with $k=3,4,5,6$ and $X \in \mathcal{P}_{0}=\{(i, j) / 20,1 \leq i, j \leq 10\}$. Line $i$ and column $j$ correspond to the pole $X=\left(\frac{i}{20}, \frac{j}{20}\right)$. As mentioned in (4.1), the fourth and fifth eigenvalues, $\lambda_{4}\left(\dot{\Omega}_{X}^{\mathcal{R}}\right)$ and $\lambda_{5}\left(\dot{\Omega}_{X}^{\mathcal{R}}\right)$, are equal to the second eigenvalue of the square, hence the associated eigenfunctions of the Dirichlet-Laplacian on the square have two nodal domains and those of the Dirichlet-Laplacian on the double covering $\dot{\Omega}_{X}^{\mathcal{R}}$ have three or four nodal domains according to the location of $X$. We do not represent them here. We have observed that $\lambda_{4}^{\mathbf{A B} X}$ equals $\lambda_{7}\left(\dot{\Omega}_{X}^{\mathcal{R}}\right)$ when $X$ is close to the center $C$ and $\lambda_{8}\left(\dot{\Omega}_{X}^{\mathcal{R}}\right)$ for $X$ close to the corner $X=(0,0)$. The transition between these two eigenvalues is represented in Figure A2 by the separation line (dotted line). Similarly, $\lambda_{5}^{A B X}$ equals $\lambda_{9}\left(\dot{\Omega}_{X}^{\mathcal{R}}\right)$ or $\lambda_{11}\left(\dot{\Omega}_{X}^{\mathcal{R}}\right)$ for $X$ close to the center $X=C$ and the transition is represented in Figure A3 by the separation line (dotted line).

We verify the property proved in [10] that from the pole $X$ only an odd number of lines can start for the nodal set corresponding to an $\mathbf{A B} X$-eigenfunction. 


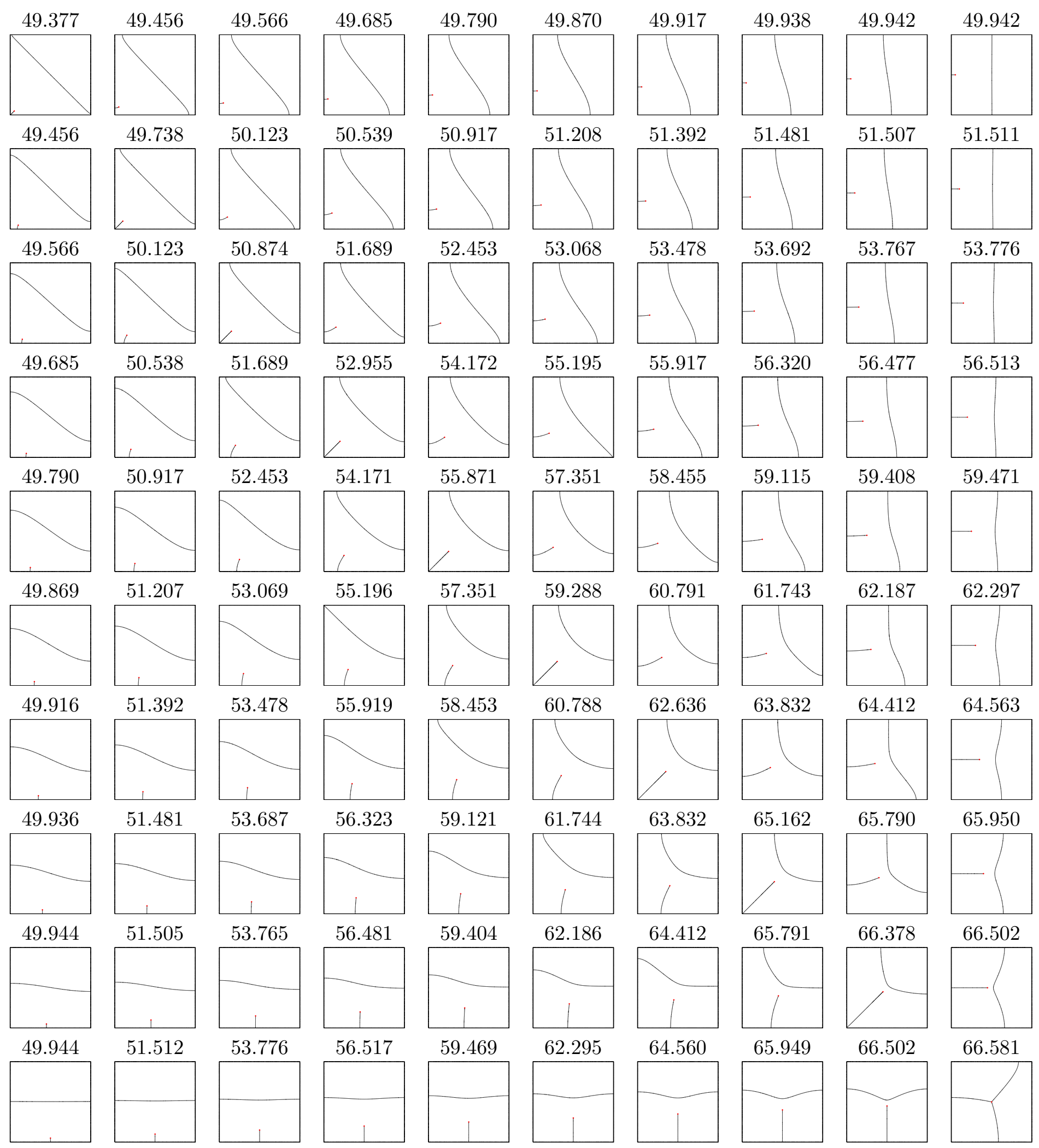

Figure A1: Third eigenvalue $\lambda_{3}^{\mathbf{A B}}\left(\dot{\Omega}_{X}\right)$. 


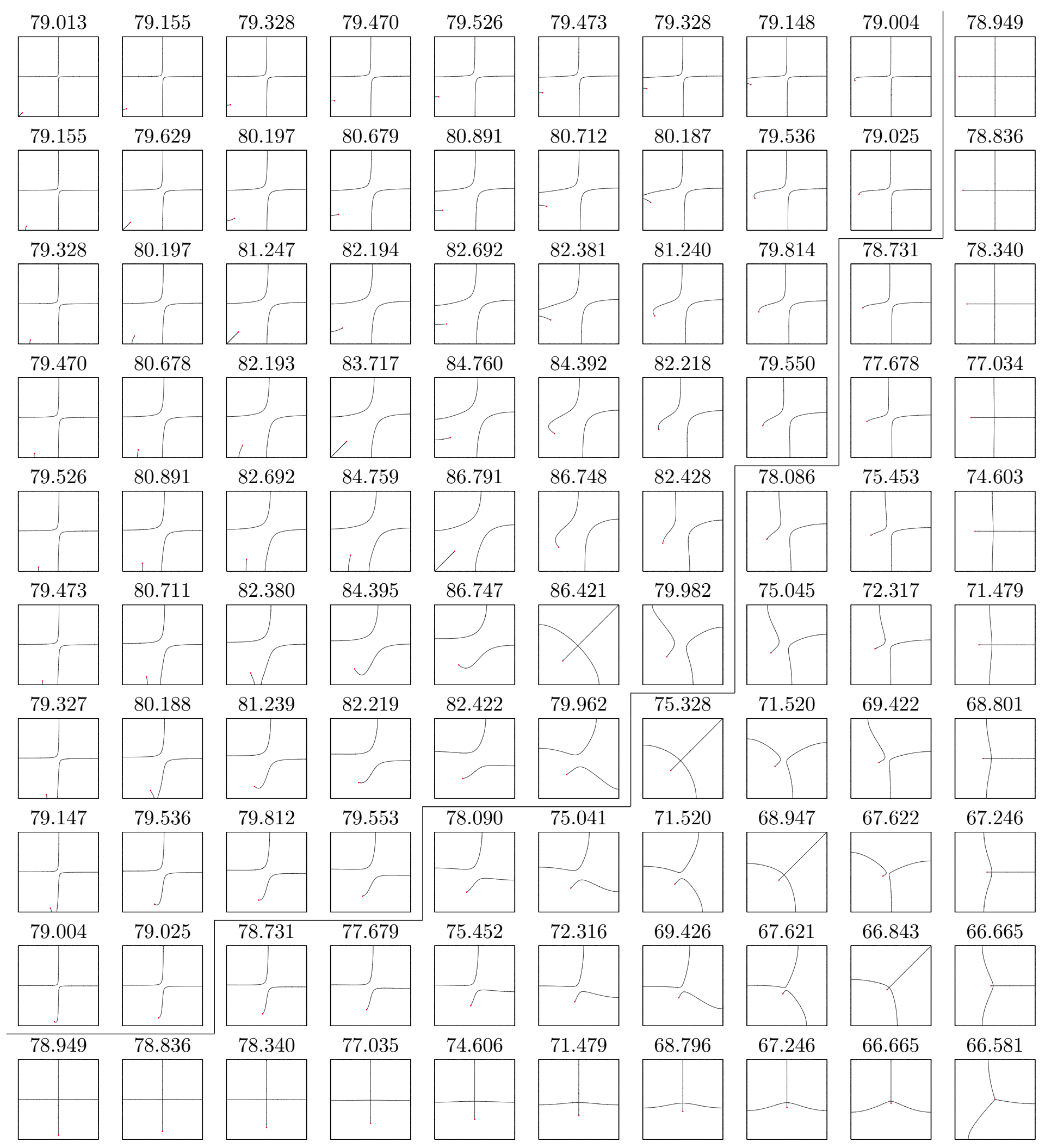

Figure A2: Fourth eigenvalue $\lambda_{4}^{\mathbf{A B}}\left(\dot{\Omega}_{X}\right)$. 


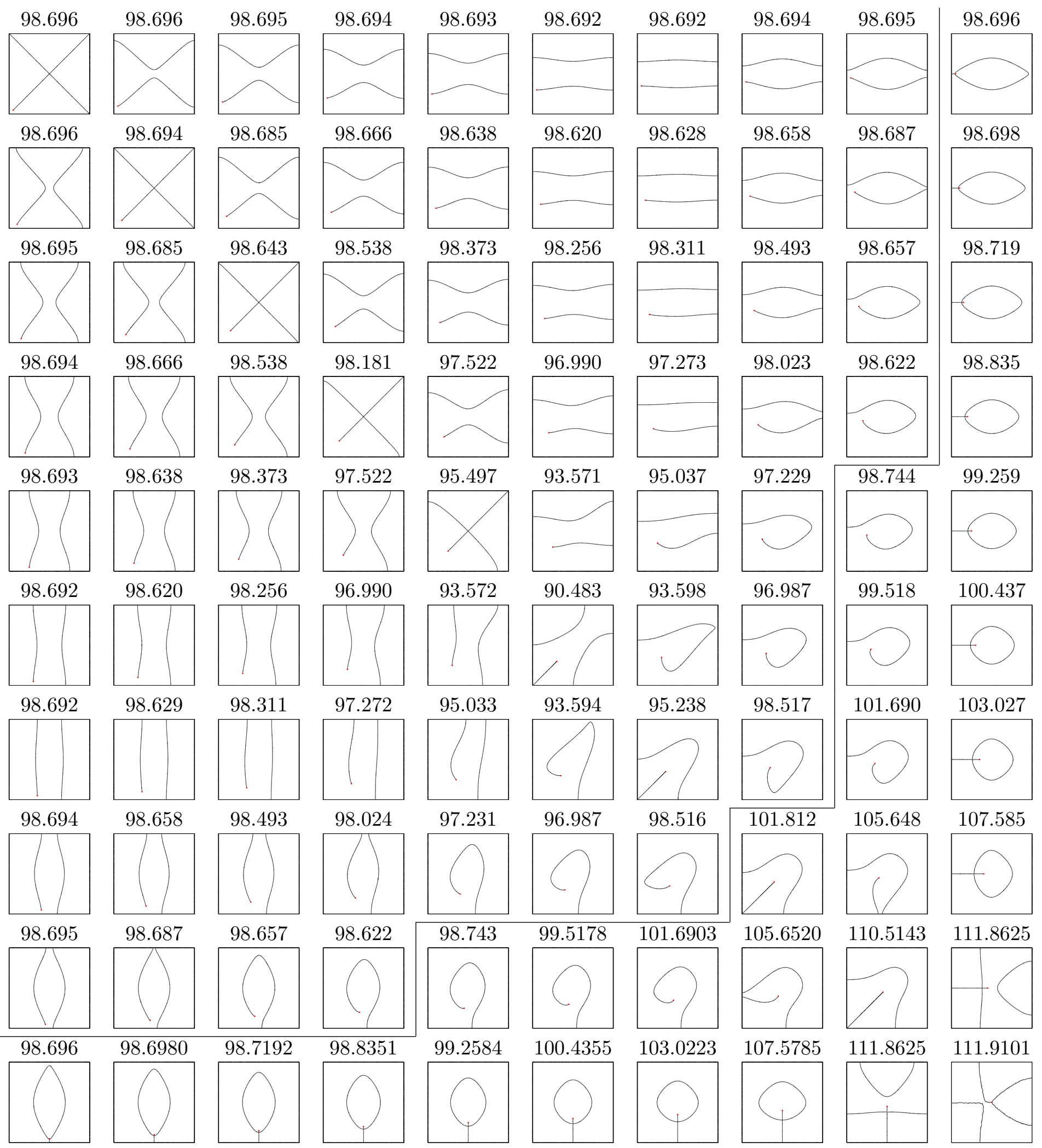

Figure A3: Fifth eigenvalue $\lambda_{5}^{\mathbf{A B}}\left(\dot{\Omega}_{X}\right)$. 


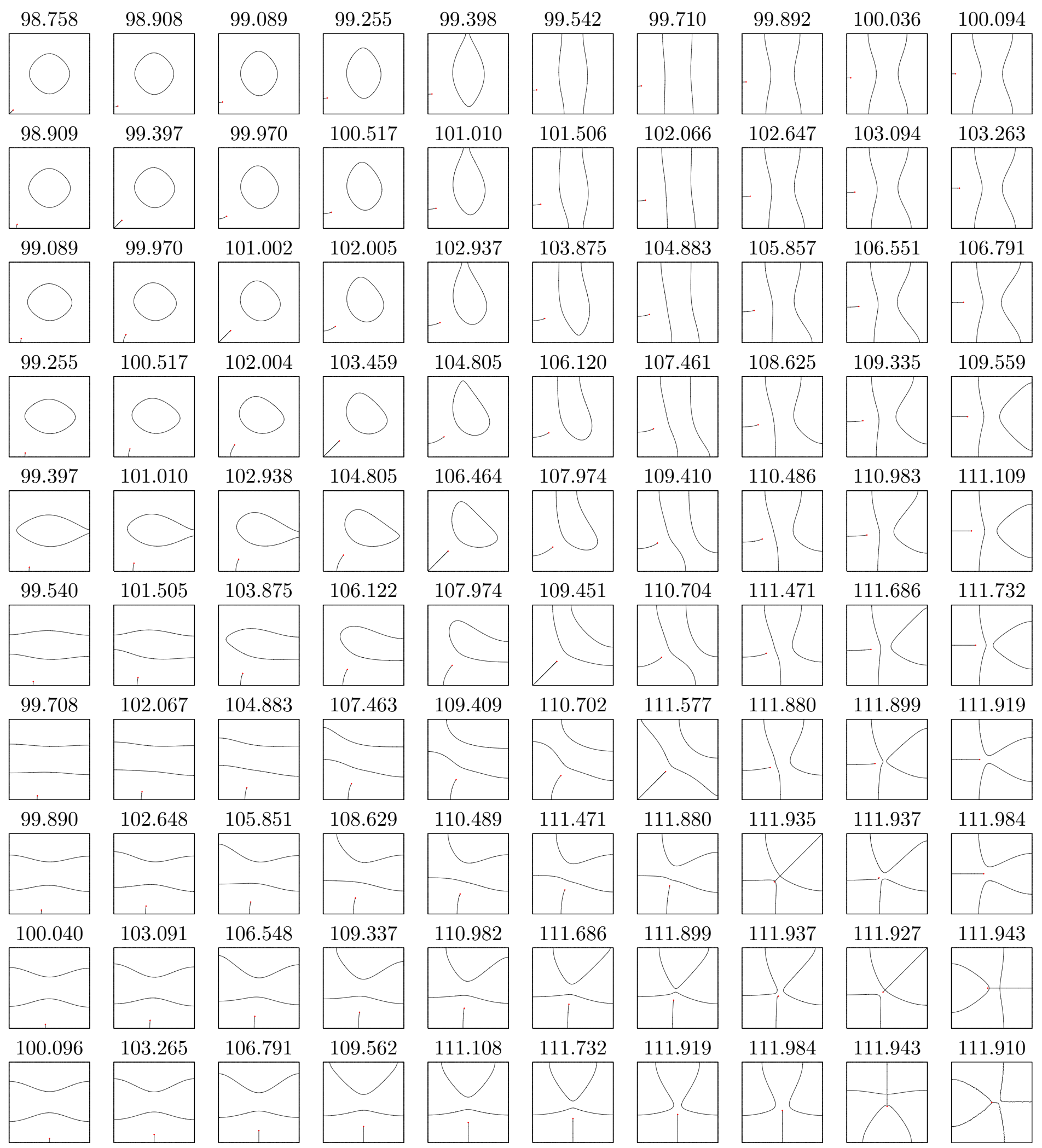

Figure A4: Sixth eigenvalue $\lambda_{6}^{\mathbf{A B}}\left(\dot{\Omega}_{X}\right)$. 